\title{
Information needs in people with diabetes mellitus: a systematic review
}

\author{
Lisa Biernatzki ${ }^{{ }^{*}+}$, Silke Kuske ${ }^{1,2 \dagger}$, Jutta Genz ${ }^{2}$, Michaela Ritschel ${ }^{1,2}$, Astrid Stephan ${ }^{1}$, Christina Bächle ${ }^{1,2}$, \\ Sigrid Droste ${ }^{1 \wedge}$, Sandra Grobosch ${ }^{1,2}$, Nicole Ernstmann ${ }^{3}$, Nadja Chernyak ${ }^{1,2}$ and Andrea Icks ${ }^{1,2,4}$
}

\begin{abstract}
Background: The purpose of this study was to identify and analyse currently available knowledge on information needs of people with diabetes mellitus, also considering possible differences between subgroups and associated factors.

Methods: Twelve databases including MEDLINE, EMBASE and the Cochrane Library were searched up until June 2015. Publications that addressed self-reported information needs of people with diabetes mellitus were included. Each study was assessed by using critical appraisal tools, e.g. from the UK National Institute for Health and Care Excellence. Extraction and content analysis were performed systematically.

Results: In total, 1993 publications were identified and 26 were finally included. Nine main categories of information needs were identified, including 'treatment-process', 'course of disease', 'abnormalities of glucose metabolism' and 'diabetes through the life cycle'. Differences between patient subgroups, such as type of diabetes or age, were sparsely analysed. Some studies analysed associations between information needs and factors such as participation preferences or information seeking. They found, for example, that information needs on social support or life tasks were associated with information seeking in Internet forums.
\end{abstract}

Conclusion: Information needs in people with diabetes mellitus, appear to be high, yet poorly investigated. Research is needed regarding differences between diverse diabetes populations, including gender aspects or changes in information needs during the disease course.

Systematic review registration: The review protocol has been registered at Prospero (CRD42015029610).

Keywords: Diabetes, Information needs, Information-seeking behaviour, Systematic review

\section{Background}

Diabetes mellitus (DM) is a highly prevalent chronic disorder. People with DM have to perform comprehensive self-management interventions to achieve good diabetes control [1]. In order to make adequate decisions concerning their illness, a sufficient level of disease-related information is required [2]. In fact, people with DM communicate a particularly high need for information, higher than people with cancer or cardiovascular diseases, for example [3, 4]. However, it seems that people with DM do not feel adequately informed about their

\footnotetext{
* Correspondence: lisa-biernatzki@gmx.de

${ }^{\dagger}$ Equal contributors

Deceased

${ }^{1}$ Institute for Health Services Research and Health Economics, Centre for Health and Society, Faculty of Medicine, Heinrich Heine University, Moorenstraße 5, 40225 Düsseldorf, Germany

Full list of author information is available at the end of the article
}

condition or regarding medication use [3]. Although the importance of an appropriate needs-driven information supply is unquestioned, and a large amount of diabetes information exists, there seems to be limited knowledge about information needs (IN) of people with DM considering different patient subgroups, as well as IN of people with DM in phases of the disease that may affect the need for certain information [5-7]. To provide needsdriven information, deeper insight into the perspectives of people with DM is urgently needed. This is particularly true since it has been suggested that information supply, self-management and health outcomes could be improved if more were known about the perspectives and needs of those concerned $[8,9]$.

This systematic review aims to identify and analyse currently available knowledge on the IN of people with 
DM, also considering possible differences between subgroups and associated factors.

\section{Methods}

This systematic review was performed in line with the quality requirements of the PRISMA guideline (available as Additional file 1) [10]. The review protocol has been registered at PROSPERO (CRD42015029610).

As stated in the review protocol, we searched in MEDLINE, EMBASE, CINAHL, ScienceDirect, the Cochrane Library, Web of Science, PsycINFO, CCMed, ERIC and Journals@OVID, Deutsches Ärzteblatt and Karlsruher virtueller Katalog. Publications were included that had been published from the inception of each database up to June 2015 (see Appendix 1 and Appendix 2) [11] with a German or English title and abstract and a full text in any language.

\section{Search strategy}

The search strategy was set up using database-specific vocabularies (MeSH, EMTREE) and additional freetext terms (see Appendix 1 and Appendix 2) [11]. The search algorithm was crosschecked by experienced reviewers (S.K., A.S.) and piloted by comparing results of the search strategy in MEDLINE with core references that were identified by pre-search activities. Search terms for IN included 'information need', 'knowledge need', 'wish or desire of information', 'information preference' and 'request for information'. Search terms for DM included: 'diabetes', 'diabetic', 'niddm', 'iddm', 't2dm', 't1dm', 'prediabetes', 'prediabetic', 'pre-diabetes', 'pre-diabetic' and 'impaired glucose'. For further details see Appendix 1 and Appendix 2.

\section{Inclusion and exclusion criteria}

Studies that analysed self-reported IN of people with DM (any type) as a primary or secondary research aim were included. IN are defined as: 'Recognition that their (people's) knowledge is inadequate to satisfy a goal, within the context/situation that they find themselves at a specific point in the time [12].' Original qualitative, quantitative or mixed-methods studies were included. Systematic and narrative reviews, meta-analyses and qualitative meta-syntheses were also included.

Studies reporting the IN of relatives or healthcare professionals were excluded, as well as studies where relatives or healthcare professionals reported IN of people with DM. Publications without available references, letters/short reports, abstracts, editorials, comments or discussion papers were excluded.

\section{Study selection process}

Inclusion and exclusion criteria were pre-tested on 380 records and finally discussed (L.B., J.G., S.K.). Then, two reviewers (L.B., J.G.) independently selected the articles, first by title and abstract and thereafter by full text. All decisions were checked by two other reviewers (S.K., M.R.). Unclear decisions were resolved by an additional reviewer (A.I.).

Full texts were screened with the aim of identifying further original studies using backward citation tracking.

\section{Data extraction and synthesis}

A data extraction sheet was developed following the requirements of Cochrane [10].We extracted the type of information needed by people with DM and, if investigated, by different subgroups, such as type of DM and age. A content analysis was conducted, developing categories according to the topics of the review questions, in particular, to assess the reported and analysed types of information needed. Furthermore, IN-associated factors were extracted and analysed via a content analysis. Codings were developed inductively (L.B.) [13] using a coding protocol and revised critically (S.K., A.I.).

All relevant publications were described according to the following predefined categories: author, date, methods, findings, associated factors and result of the critical appraisal of the study quality. Furthermore, the main categories of IN were described, as well as those related to subgroups of people with DM and the associated factors. We describe the studies stratified for those analysing IN as a primary and a secondary outcome, as well as those with a qualitative, quantitative, or mixedmethod study design.

\section{Critical appraisal}

Each study was critically appraised separately (L.B., S.K., J.G., M.R.) by using design-specific critical appraisal tools from the UK National Institute for Health and Care Excellence (NICE) [14]. The study's quality was described as follows: ' $(++)$ All or most of the checklist criteria have been fulfilled, where they have not been fulfilled the conclusions are very unlikely to alter. $(+)$ Some of the checklist criteria have been fulfilled, where they have not been fulfilled, or not adequately described, the conclusions are unlikely to alter. (-) Few or no checklist criteria have been fulfilled and the conclusions are likely or very likely to alter.' [14]. Mixed methods were analysed by the Mixed Methods Appraisal Tool (MMAT)-Version 2011 [15]. The critical appraisal for mixed-methods studies includes whether the mixedmethods design was appropriate and whether the integration was relevant to address the research question (objective). The criteria also consider whether limitations are considered, associated with this integration, e.g. whether the divergence of qualitative and quantitative data (or results) in a triangulation design was appropriate [15]. 


\section{Results}

In total, 1993 publications were identified that had been published up to June 2015 (Fig. 1), of which 26 publications ( $n=$ 25 studies) reporting diabetes-related IN of people with DM were finally included (Table 1). The sample sizes varied from 11 to 1609 participants with DM.

Thirteen studies investigated IN as a primary outcome (primary research aim). Twelve of these studies analysed the type of information needed by people with DM $[3,8,9$, 16-25], and one study investigated the relationships between IN, diagnosis and disease [4] (Table 1). The other studies $(n=12)$ reported IN as a secondary outcome and focused predominantly on other topics, e.g. information exchange, patient experience and information sources [6, 26-36]. Only two studies addressed unmet IN $[3,17]$, although these were often mentioned in the discussion of the publications $[3,8,17,24,36]$. The quality of information provided, in terms of readability and comprehensiveness, was not analysed in the included studies.

Altogether, we identified 14 qualitative studies, six quantitative studies and five mixed-method studies. Four different methods were reported throughout: interviews $[3,4,8,9,17,19,23]$, group methods [16, 20, 24], surveys using written questionnaires [3, 21, 25], and website evaluation $[18,22]$. Most of the instruments were specifically developed for the respective study, and five were validated regarding their understandability, suitability and feasibility $[3,4,8,9,21,23]$. Validation regarding the validity and reliability of the instruments was not reported.

Eighteen studies used open questions' (e.g. 'Can you give an example of the type of information you have searched for?') $[4,6,8,9,16,17,19,20,23-31,33,36]$, while four studies used closed questions' $[3,8,9,21,25]$. Two of the included studies analysed online posts $[18,32]$, and one examined e-mail requests [22], whereas 12 of them asked for IN focusing on one special topic, e.g. 'pregnancy planning and childbearing' [21, 22, 29], 'information on medication' [4, 16, 17], 'oral health' [28], 'fitness and nutrition' [30, 33], 'quality of diabetes care' [36], 'automatic telephone outreach' [26] and 'blood pressure control' [6]. Some studies used more than one approach.

The critical appraisal showed that three of the 25 identified studies met all or most of the NICE checklist criteria. The other studies fulfilled some $(n=14)$ or a few criteria $(n=8)$. It was noticeable that within the qualitative studies, most of the criteria were fulfilled but eight of 14 studies did not describe the role of the researcher sufficiently, and six gave no indications concerning ethical approval. None of the included quantitative studies reported how selection bias was minimised, and included studies using mixed-method design reported little about the quantitative part of their study

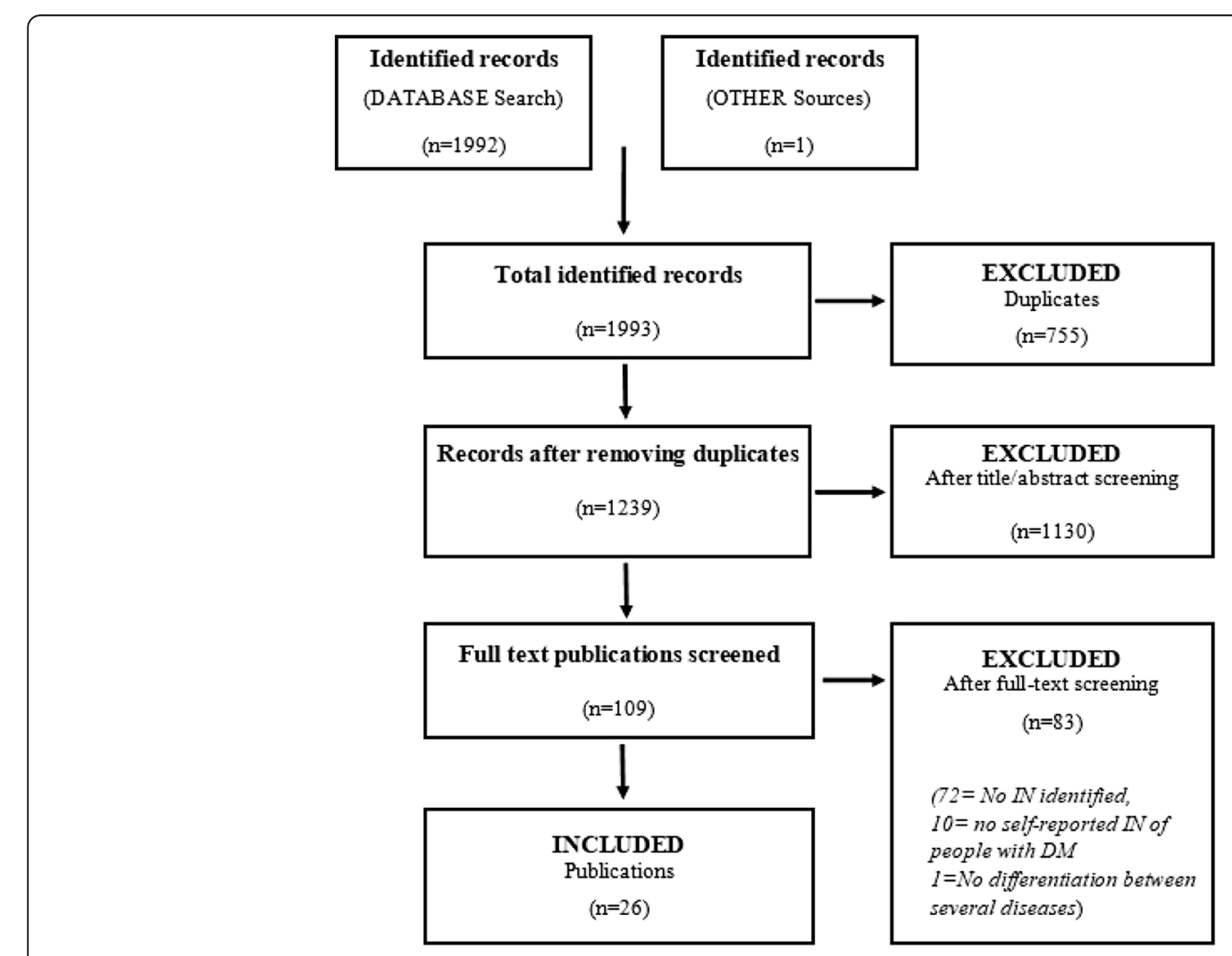

Fig. 1 Study Selection Process 
Biernatzki et al. Systematic Reviews (2018) 7:27

Page 4 of 21

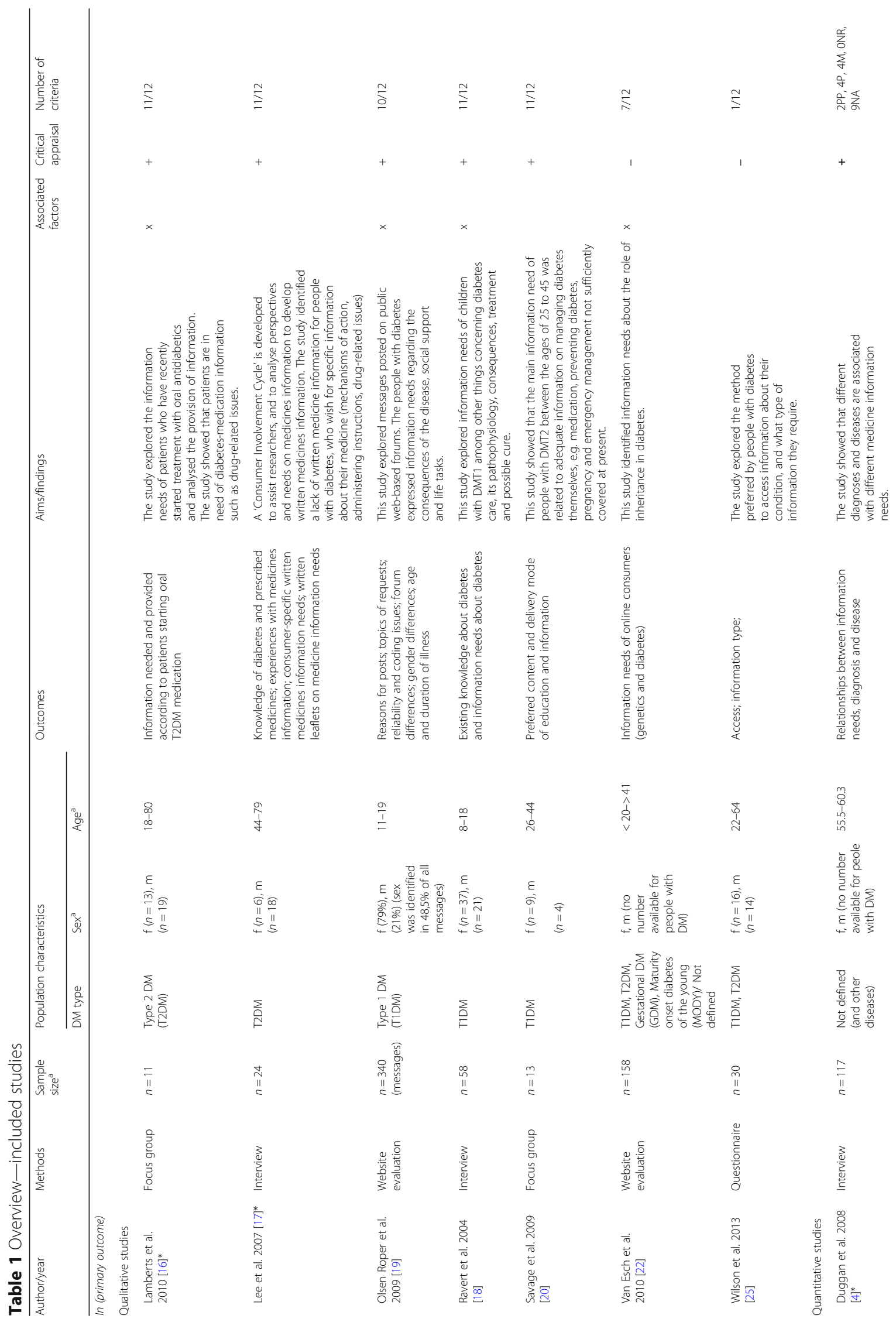


Biernatzki et al. Systematic Reviews (2018) 7:27

Page 5 of 21

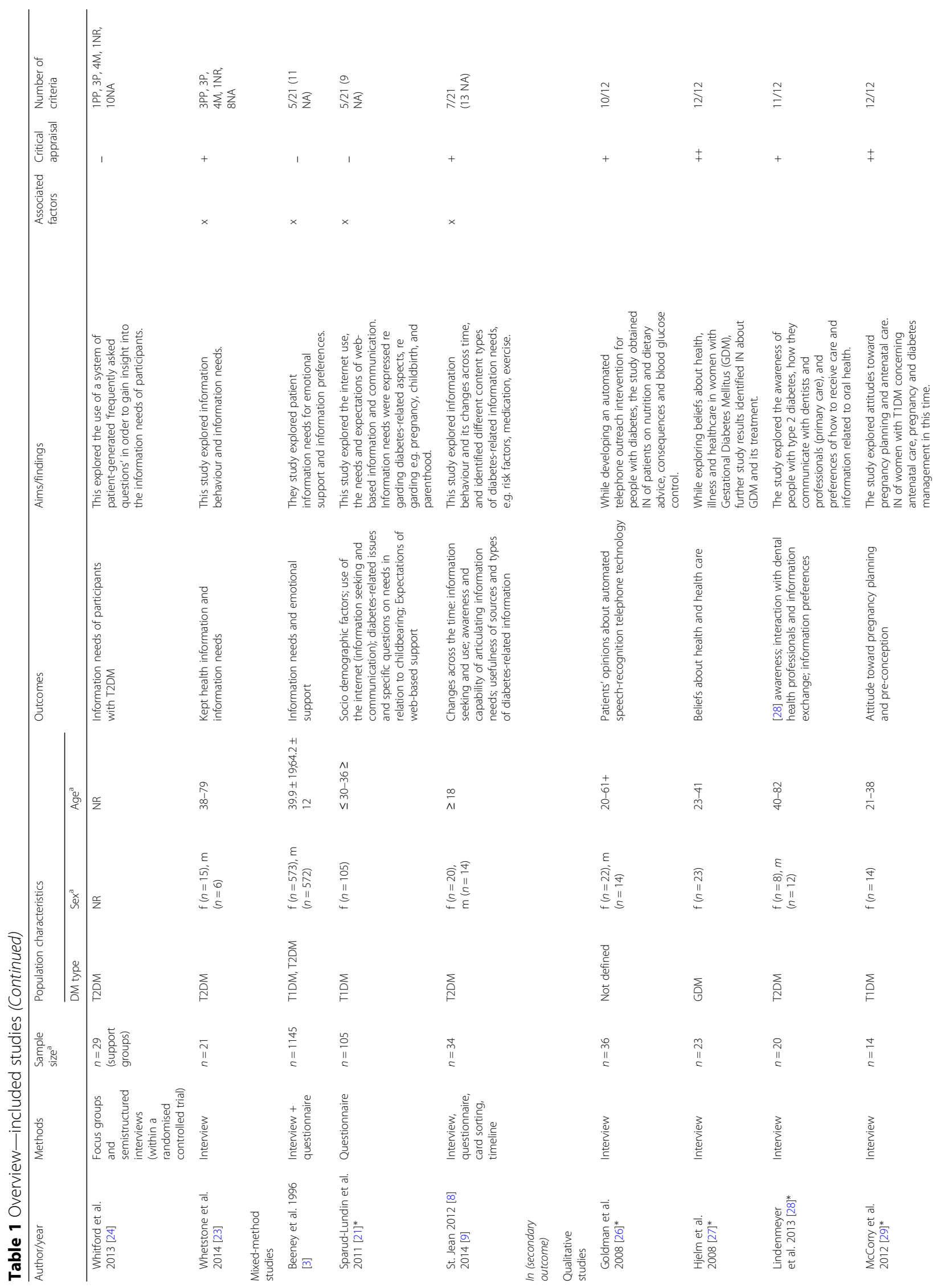




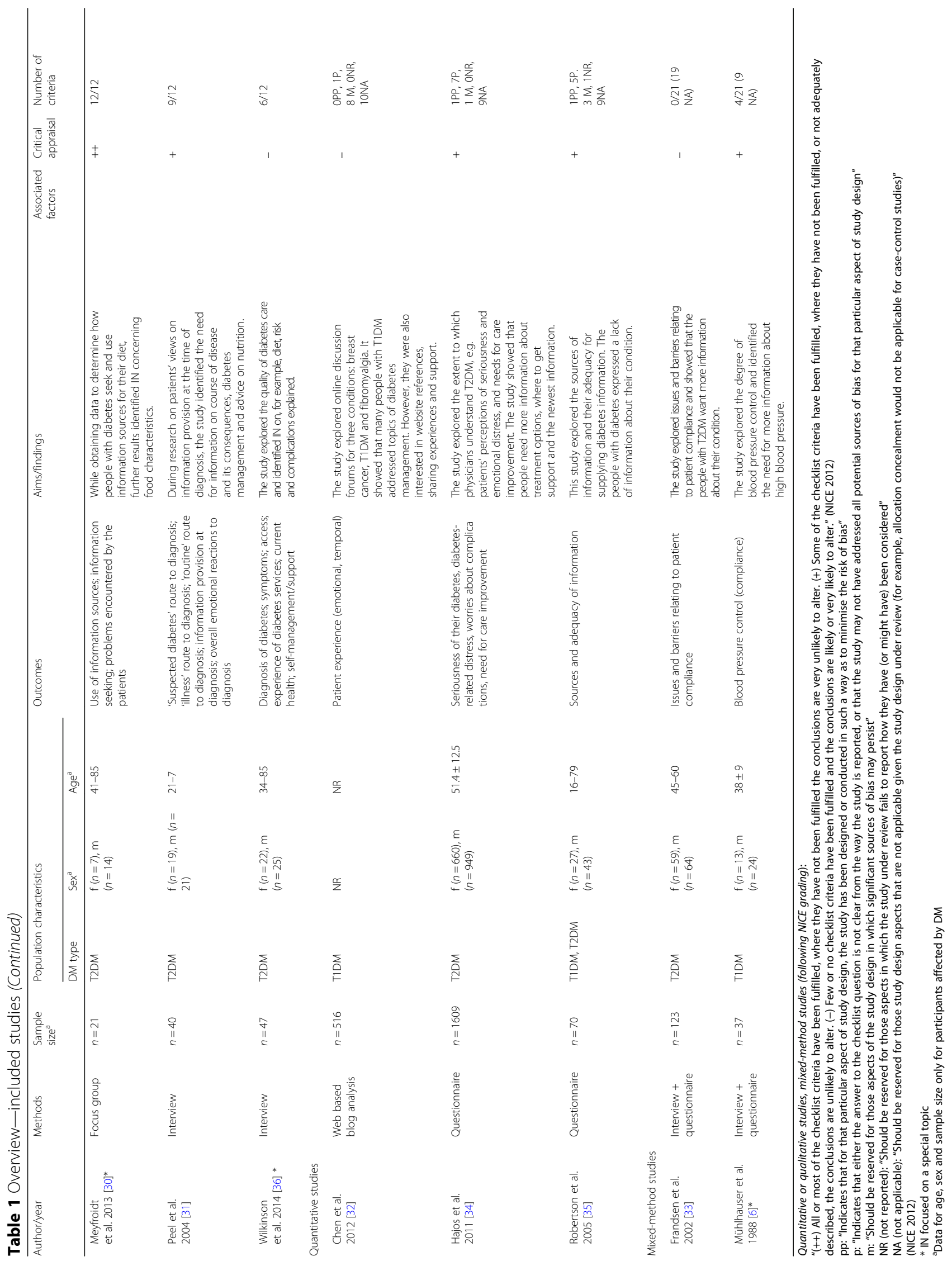


design. The results of the critical appraisals are shown in Table 1.

\section{Content of information needs}

The content analysis (Table 2) identified nine main types of IN of people with DM and 28 subtypes. The main categories are 'treatment-process', 'course of disease', 'abnormalities of glucose metabolism,' 'diabetes through the life cycle, 'pathophysiology of diabetes,' 'research', 'coping,' 'support', and 'prevention'. IN on the 'treatment process' were reported most frequently throughout the studies, in particular, 'medication' $(n=12)$, 'diabetes self-management' ( $n$ $=11)$ and 'nutrition' $(n=11)$. IN regarding the 'course of disease' were the second highest reported, particularly 'consequences of diabetes' $(n=16)$, e.g. consequences concerning physical health, lifestyle and social life. Only four studies reported IN on 'coping' and 'support', and two reported on 'prevention'.

\section{Information needs in subgroups and factors associated to IN} Specific comparisons between subgroups or analysis of associated factors using, for instance, regression models were performed minimally in the identified studies. Only one mixed-method study made a comparison between type 1 DM (T1DM) and type 2 DM (T2DM) [3]. Hence, we could only try to find subgroup-specific IN from studies that addressed certain groups as people with T1DM or T2DM or women with gestational DM (GDM). Only two studies investigated related factors such as, for example, socio-economic status $[4,8,9]$. Duggan et al. was the only study that performed quantitative statistics. The authors found, for example, that higher socio-economic status was positively correlated with the need for drug information [4]. More complex factors such as concepts like participation preferences or seeking behaviour were analysed more frequently than often-investigated associated factors such as age and sex, and these analyses were predominantly performed in qualitative studies, in particular in the study by St. Jean [8, 9] and Whetstone [23]. In the following, we describe the main findings.

\section{Information needs and types of diabetes}

Twelve studies (13 publications) explicitly addressed people with T2DM $[8,9,16,17,20,23,24,28,30,31$, $33,34,36]$ and six explicitly addressed people with T1DM [6, 18, 19, 21, 29, 32]. Additionally, four studies addressed different types of DM [3, 22, 25, 35], and two did not specify the type of DM [4, 26]. Finally, two studies focussed on women with GDM [22, 27] (Table 3). Only one mixed-method study made a comparison between T1DM and T2DM and showed that contents of patient-identified concerns between these subgroups are similar, apart from 'not knowing enough' (T1DM) and 'concerns about future' (T2DM) [3].

Overall, no striking differences between the IN of people with different types of DM were identified or noticeable in one certain group. Almost all the studies, with the exception of studies involving people with GDM and maturity onset diabetes of the young (MODY) [22, 27], reported IN in the categories 'treatment process', 'course of disease,' pathophysiology of diabetes,' research,' 'coping,' support' and 'prevention'. With the exception of the studies involving people with T1DM, all the studies reported IN regarding 'abnormalities of glucose metabolism'. Furthermore, 'diabetes through the life cycle' was addressed in most of the studies, but not in the study including people with MODY.

\section{Information needs and age}

Overall, only a few differences of IN between people with DM in different age groups were identified in the studies; however, some were reported. Young people with T1DM were particularly interested in 'diabetes through the life cycle', e.g. 'pregnancy'. There were two populations of young people identified: children with mean age 10 to 13 [19] and adolescents and young adults aged between 14 and 25 [18, 22]. Several IN were reported by both groups; however, there were also differences: the study by Olsen Roper et al. reporting IN of children between 10 and 13 years old showed that the population of children was particularly interested in the topic 'course of disease', especially 'cure' issues [19]. Additionally, they were interested in 'abnormalities of glucose metabolism', particularly 'pathogenesis' and 'aetiology' of diabetes [19]. In contrast, the population of adolescents and young adults was interested in 'diabetes through the life cycle, particularly in 'puberty' [18], 'family founding' [22] and 'pregnancy' [22].

Older people with DM were interested in 'treatment process', the 'course of disease' and the 'abnormalities of glucose metabolism'. A further differentiation in age groups, e.g. 'elderly' and 'very old', cannot be derived from the studies.

\section{Information needs and information provision as well as information seeking}

Six studies addressed IN in association with information provision and seeking $[8,9,16,18,21-23]$. General information about oral anti-hyperglycaemic medication and diabetes is provided preferably by general practitioners [16]. Furthermore, the provision of Internet-based information was recommended for the needs of childbearing women and young women, respectively [21, 22]. Information seeking in forums was associated with IN on 'social support', 'life tasks', 'factual information' and 'management information' [18]. Material items (such as 'books, news clippings, journal articles, printouts from an Internet site or notes of references that are maintained in the home') are associated 


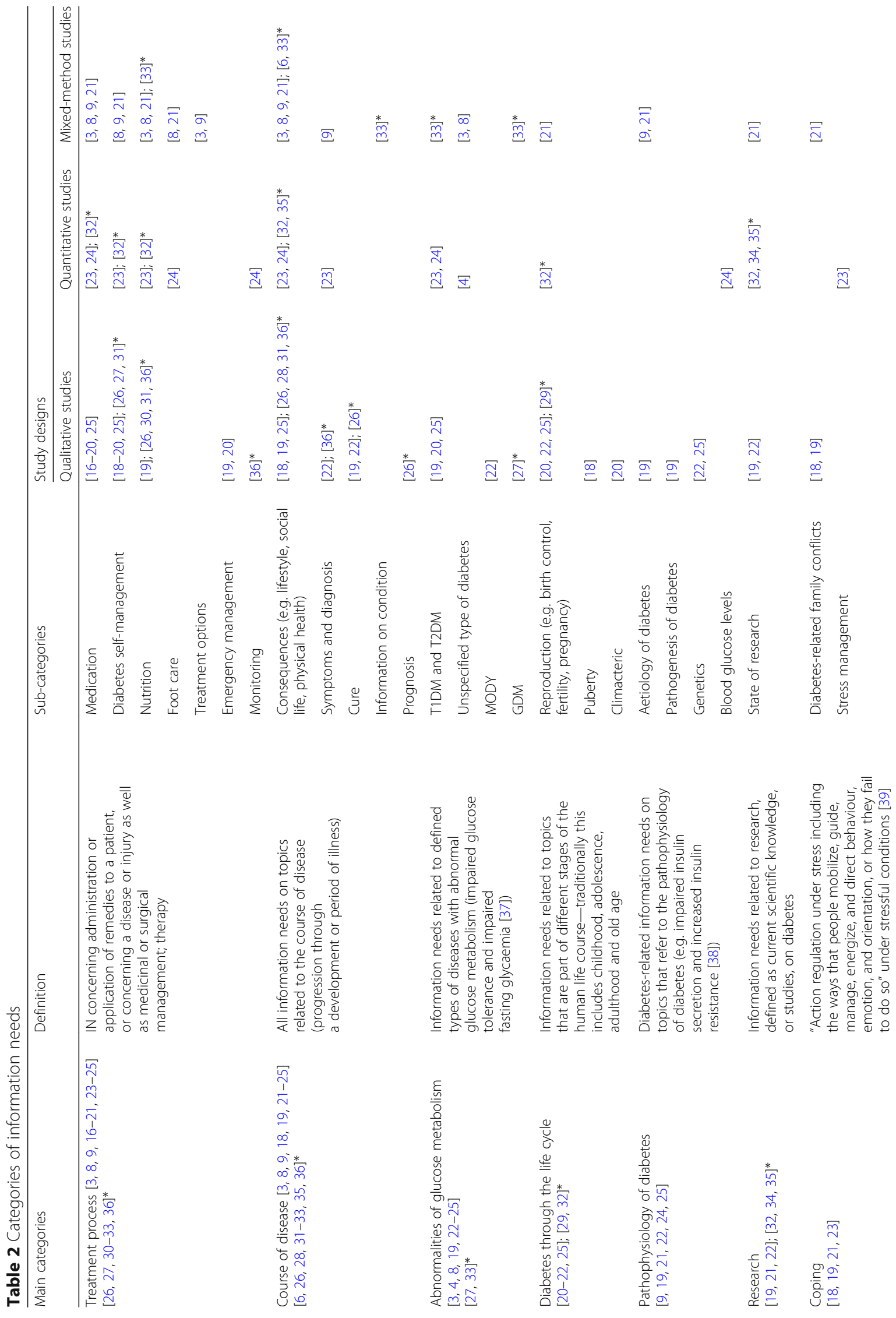




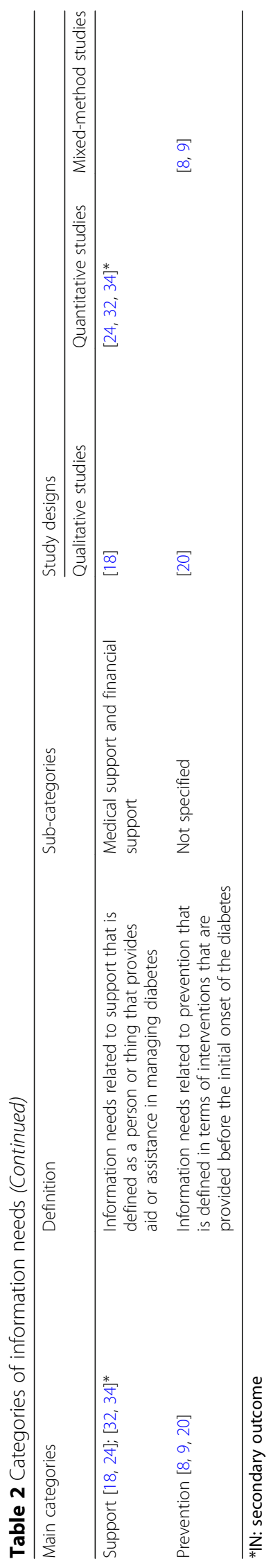




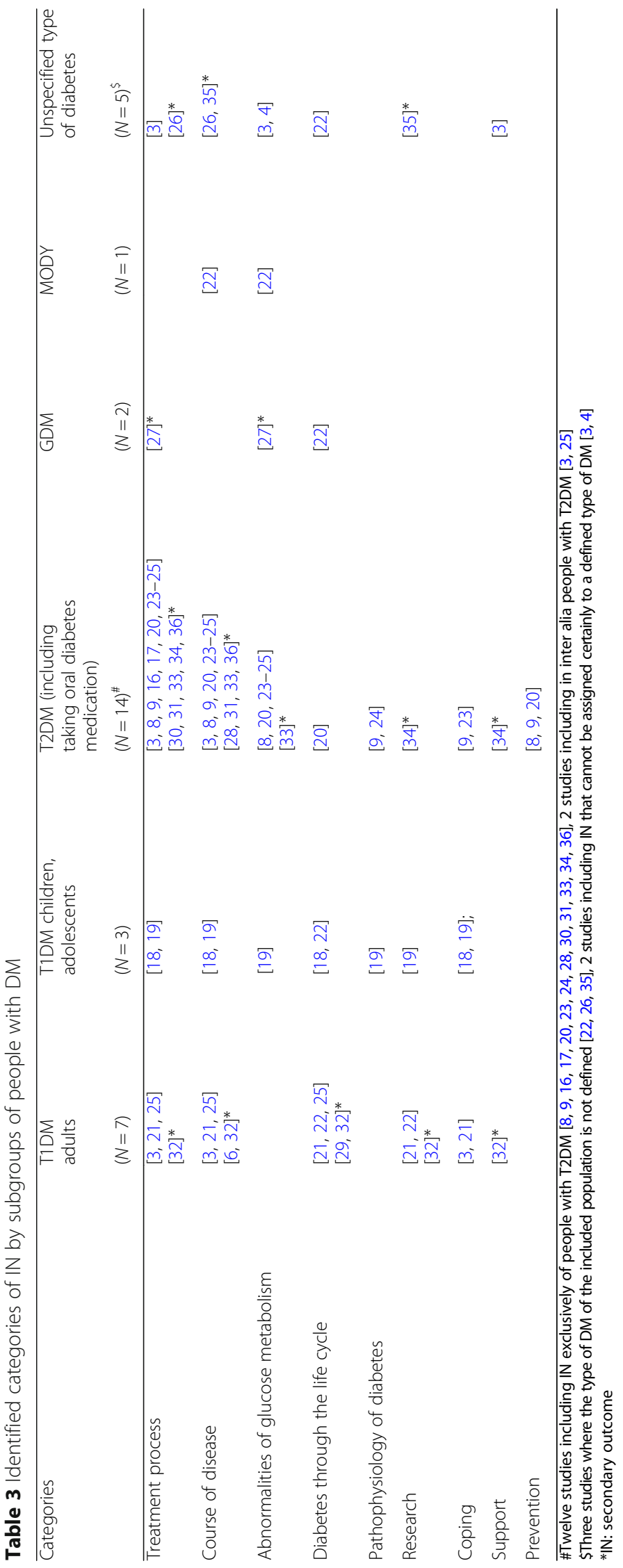


with different IN [23]. For example, IN on the topic 'nutrition' are associated with information sources such as cookbooks, hand-outs and self-selected website print-outs [23]. Generally, more frequent seeking for diabetes-related information was associated with lower ratings for the usefulness of information regarding diabetes-related complications $[8,9]$.

\section{Information and participation preferences, knowledge about and experience with diabetes}

Three studies addressed IN in association with participation preferences. All three studies analysed IN in relation to decision-making and involvement in the decision process $[3,8,9,25]$. St. Jean pointed out that an involvement in decision-making is related to higher ratings for the usefulness of information $[8,9]$.

Two studies addressed IN in association with 'knowledge' and '(feelings about) diabetes experience' $[8,9,19]$. Knowledge, diabetes experience and IN are strongly related. Therefore, both studies recommended identifying the individual level of knowledge and whether this is correct $[8,9,19]$. Furthermore, clear, optimistic, lessuncertain feelings, or support in diabetes experience were associated with different IN, e.g. 'diabetes management', 'causes of diabetes', diabetes-related complications' $[8,9]$.

\section{Stage of the disease}

One study that focused on people with DM who had recently started treatment with oral anti-hyperglycaemic drugs addressed IN in association with the stage of the disease. It pointed out that people with DM who recently started treatment with anti-hyperglycaemic drugs are in need of diabetes medication information such as drug-related issues [16].

\section{Discussion}

This is the first systematic review of studies dealing with IN of people with DM. We identified 25 studies (26 publications). This is a limited number compared with, say, cancer, where a large number of quantitative and qualitative IN studies $(n=112)$ already exist [37]. This is surprising, since it is known that people with DM have a higher or similarly high need for information compared with people with other chronic diseases [3, 4]. Looking for the content of IN, it was comparable to those found in people with cancer, such as 'prognosis of disease', 'diagnostic tests', 'treatment', 'self-care', and 'emotional and psychological needs' [37]. However, Duggan et al. found that people with DM have a higher need for information about drugs than people with cancer or cardiovascular disease [4].

It would be interesting to look for differences between people with diabetes and cancer or other chronic diseases in more detail.
Besides the low number of studies, it became obvious that differences between patient groups such as male and female patients, different age groups or types of diabetes have not been analysed so far. Factors associated with IN are rarely investigated. In cases in which analysis was performed, mainly the more complex factors such as participation preferences or information seeking were investigated, and it was particularly done in qualitative studies, which were highly heterogeneous. Also, changes in IN during the course of the disease are poorly investigated, although they may be expected.

Instruments for collecting IN data from individuals with DM have not been validated. Similar results were identified in cancer studies and showed that only a minority of instruments for the collection of IN are validated [37].

\section{Implications for research}

There is a need for research on several levels. First, compared with other chronic diseases, there is a huge lack of studies addressing IN of people with $\mathrm{DM}$, although DM is one of the largest public health issues [38]. Due to the low number of studies dealing with unmet needs, and considering the relevance of this topic mentioned in the studies, there is a need for further research. Second, differences between the types of DM populations including gender aspects and patient characteristics should be analysed. Third, there is a need for research to show associations of variables with IN and to amend the findings from qualitative studies via qualitative analyses. Available knowledge about the IN and associated factors and concepts can be used in targeted counselling and to strengthen the health literacy of people with DM. Finally, methods and instruments should be further developed against a theoretical background and validated.

\section{Limitations}

We conducted a comprehensive and sensitive search that was also pre-tested. The study selection and critical appraisal were performed by two reviewers. Two other reviewers checked all the decisions. The critical appraisals were performed for each study design. However, there were still difficulties to provide a clear decision based on the reports.

The identified studies have several limitations: only three qualitative studies met all the quality criteria. None of the quantitative studies met all the criteria. The comparability of the IN categories is restricted because the IN were collected by different study designs. Some of them were collected in the context of a specific health-related topic or by using different methods or instruments. 


\section{Conclusion}

There is a limited number of studies analysing IN in $\mathrm{DM}$, and there is a low number of studies investigating differences between subgroups of DM populations, including gender aspects or changes of information needs during the disease. This should be further investigated.

\section{Appendix 1 \\ Search terms (26/06/2014) [11] \\ MEDLINE (OVID)}

Database: MEDLINE (1946-2014)

Research period: unlimited

\begin{tabular}{|c|c|c|}
\hline $\begin{array}{l}\text { Search } \\
\text { step }\end{array}$ & Hits & Search \\
\hline 1 & 702 & EXP INFORMATION SEEKING BEHAVIOR/ \\
\hline 2 & 1718 & EXP INFORMATION LITERACY/ \\
\hline 3 & 3397 & EXP CONSUMER HEALTH INFORMATION/ \\
\hline 4 & 70,372 & EXP PATIENT EDUCATION AS TOPIC/ \\
\hline 5 & 600 & EXP HEALTH COMMUNICATION/ \\
\hline 6 & 1279 & 1 OR 5 \\
\hline 7 & 34 & $\begin{array}{l}6 \text { AND (diabetes OR diabetic OR niddm OR iddm OR } \\
\text { t2 dm OR } 11 \mathrm{dm} \text { OR prediabetes OR prediabetic OR } \\
\text { pre-diabetes OR pre-diabetic OR impaired } \\
\text { glucose).ti,ab. }\end{array}$ \\
\hline 8 & 3488 & 2 OR 3 \\
\hline 9 & 80 & $\begin{array}{l}8 \text { AND (diabetes OR diabetic OR niddm OR iddm OR } \\
\text { t2 dm OR t1 dm OR prediabetes OR prediabetic OR } \\
\text { pre-diabetes OR pre-diabetic OR impaired glucose).ti,ab. } \\
\text { AND information.ti,ab. }\end{array}$ \\
\hline 10 & 107 & $\begin{array}{l}4 \text { AND ((diabetes OR diabetic OR niddm OR iddm OR } \\
\text { t2 dm OR t1 dm OR prediabetes OR prediabetic OR } \\
\text { pre-diabetes OR pre-diabetic OR impaired glucose).ti,ab. } \\
\text { AND ((interest* OR need OR needs OR question* OR } \\
\text { asking OR ask OR seek* OR search* OR demand OR } \\
\text { desire OR request* OR call OR requirement OR } \\
\text { requiring OR preference* OR wish OR wishes OR } \\
\text { provision* OR expectation*) ADJ5 information)).ti,ab. }\end{array}$ \\
\hline 11 & 45 & $\begin{array}{l}\text { (information needs AND (diabetes OR diabetic OR } \\
\text { niddm OR iddm OR t2 dm OR t1 dm OR prediabetes } \\
\text { OR prediabetic OR pre-diabetes OR pre-diabetic OR } \\
\text { impaired glucose)).ti,ab. }\end{array}$ \\
\hline 12 & 3935 & $\begin{array}{l}\text { ((education OR communication) ADJ2 need OR needs } \\
\left.\text { OR preference }{ }^{*}\right) \text { ).ti,ab. }\end{array}$ \\
\hline 13 & 85 & $\begin{array}{l}12 \text { AND (diabetes OR diabetic OR prediabetes OR } \\
\text { prediabetic OR pre-diabetes OR pre-diabetic OR } \\
\text { impaired glucose).ti. }\end{array}$ \\
\hline 14 & 1721 & *PATIENT PREFERENCE/ \\
\hline 15 & 13 & $\begin{array}{l}14 \text { AND (information OR education OR } \\
\text { communication).ti,ab. AND (diabetes OR diabetic OR } \\
\text { prediabetes OR prediabetic OR pre-diabetes OR } \\
\text { pre-diabetic OR impaired glucose).ti,ab. }\end{array}$ \\
\hline 16 & 9730 & $\begin{array}{l}\text { ((patient OR patient-centered) ADJ1 (need OR needs } \\
\text { OR seek* OR search* OR demand OR desire OR } \\
\text { preference* OR wish OR wishes OR provision* OR } \\
\text { expectation*)).ti,ab. }\end{array}$ \\
\hline
\end{tabular}

(Continued)

$1734 \quad 16$ AND (diabetes OR diabetic OR prediabetes OR prediabetic OR pre-diabetes OR pre-diabetic OR impaired glucose).ti. AND (need OR needs OR preference* ${ }^{*}$.ti.

18214 AND (facilitat* OR barrier* OR pitfall*).ti. AND (diabetes OR diabetic OR prediabetes OR prediabetic OR pre-diabetes OR pre-diabetic OR impaired glucose).ti,ab.

19941 (((information OR education OR communication) ADJ3 (interest* OR need OR needs OR question* OR asking OR ask OR seek* OR search* OR demand OR desire OR request* $O R$ call $O R$ requirement $O R$ requiring $O R$ preference* OR wish OR wishes OR provision* OR expectation*)) AND (diabetes OR diabetic OR prediabetes OR prediabetic OR pre-diabetes OR pre-diabetic OR impaired glucose)).ti,ab.

2017819 AND (interest* OR need* OR question* OR ask* OR talk* OR online communi* OR message ${ }^{*}$ OR seek* OR search* OR demand OR desire OR request* OR call OR requir* OR preference* OR wish* OR perception* OR provision* OR expectation* OR facilitat* OR barrier* OR pitfall*).ti.

214014 (information AND (interest* OR need OR needs OR question* OR asking OR ask OR seek* OR search* OR demand OR desire OR request* OR call OR requirement $O R$ requiring $O R$ preference $\left.{ }^{*}\right)$ ).ti.

225321 AND (diabetes OR diabetic OR prediabetes OR prediabetic OR pre-diabetes OR pre-diabetic OR impaired glucose).ti,ab.

235 ((identify* ADJ2 (interest* OR need OR needs OR requirement $O R$ preference $\left.{ }^{*}\right)$ AND (diabetes OR diabetic OR prediabetes OR prediabetic OR pre-diabetes OR pre-diabetic OR impaired glucose)).ti.

2419 (support* AND (interest* OR need OR needs OR requirement $O R$ preference $\left.{ }^{*}\right)$ ).ti. AND (diabetes OR diabetic OR prediabetes OR prediabetic OR prediabetes OR pre-diabetic OR impaired glucose).ti,ab.

$25497 \quad 7$ OR 9 OR 10 OR 11 OR 13 OR 15 OR 17 OR 18 OR 20 OR 22 OR 23 OR 24

\section{EMBASE (OVID)}

Database: EMBASE (1974-2014)

Research period: unlimited

\begin{tabular}{lll}
\hline $\begin{array}{l}\text { Search } \\
\text { step }\end{array}$ & Hits & Search \\
1 & 989 & EXP INFORMATION SEEKING/ \\
2 & 187 & EXP INFORMATION LITERACY/ \\
3 & 2226 & EXP CONSUMER HEALTH INFORMATION/ \\
4 & 23,953 & *PATIENT EDUCATION/ \\
5 & 7326 & *MEDICAL INFORMATION/ \\
6 & 72 & 1 AND 5 \\
7 & 2 & $\begin{array}{l}\text { 6 AND (diabetes OR diabetic OR prediabetes OR } \\
\text { prediabetic OR pre-diabetes OR pre-diabetic OR }\end{array}$ \\
8 & & impaired glucose).ti,ab. \\
& 1164 & 1 OR 2
\end{tabular}




\begin{tabular}{|c|c|c|}
\hline 9 & 20 & $\begin{array}{l}8 \text { AND (diabetes OR diabetic OR prediabetes OR } \\
\text { prediabetic OR pre-diabetes OR pre-diabetic OR } \\
\text { impaired glucose).ti,ab. }\end{array}$ \\
\hline 10 & 36 & $\begin{array}{l}3 \text { AND (diabetes OR diabetic OR prediabetes OR } \\
\text { prediabetic OR pre-diabetes OR pre-diabetic OR } \\
\text { impaired glucose).ti,ab. AND (interest* OR need OR } \\
\text { needs OR question* OR asking OR ask OR seek* OR } \\
\text { search* OR demand OR desire OR request* OR call } \\
\text { OR requirement OR requiring OR preference* OR wish } \\
\text { OR wishes OR provision* OR expectation*).ti,ab. }\end{array}$ \\
\hline 11 & 65 & $\begin{array}{l}5 \text { AND (diabetes OR diabetic OR prediabetes OR } \\
\text { prediabetic OR pre-diabetes OR pre-diabetic OR } \\
\text { impaired glucose).ti,ab. AND (interest* OR need OR } \\
\text { needs OR question* OR asking OR ask OR seek* OR } \\
\text { search* OR demand OR desire OR request* OR call } \\
\text { OR requirement OR requiring OR preference* OR wish } \\
\text { OR wishes OR provision* OR expectation*).ti,ab. }\end{array}$ \\
\hline 12 & 100 & 10 OR 11 \\
\hline 13 & 24 & $\begin{array}{l}12 \text { AND ((information OR education OR support) } \\
\text { ADJ2 (interest* OR need OR needs OR question* OR } \\
\text { asking OR ask OR seek* OR search* OR demand OR } \\
\text { desire OR request* OR call OR requirement OR } \\
\text { requiring OR preference* OR wish OR wishes OR } \\
\text { provision* OR expectation*)).ti,ab. }\end{array}$ \\
\hline 14 & 43 & $\begin{array}{l}4 \text { AND ((diabetes OR diabetic OR niddm OR iddm OR } \\
\text { t2 dm OR t1 dm OR prediabetes OR prediabetic OR } \\
\text { pre-diabetes OR pre-diabetic OR impaired glucose). } \\
\text { ti,ab. AND ((interest* OR need OR needs OR question* } \\
\text { OR asking OR ask OR seek* OR search* OR demand } \\
\text { OR desire OR request* OR call OR requirement OR } \\
\text { requiring OR preference* OR wish OR wishes OR } \\
\text { provision* OR expectation*) ADJ5 information)).ti,ab. }\end{array}$ \\
\hline 15 & 65 & $\begin{array}{l}\text { (information needs AND (diabetes OR diabetic OR } \\
\text { niddm OR iddm OR t } 2 \mathrm{dm} \text { OR } \mathrm{t} 1 \mathrm{dm} \text { OR prediabetes } \\
\text { OR prediabetic OR pre-diabetes OR pre-diabetic OR } \\
\text { impaired glucose).ti,ab. }\end{array}$ \\
\hline
\end{tabular}

164941 ((education OR communication) ADJ2 (need OR needs OR preference*)).ti,ab.

$17127 \quad 16$ AND (diabetes OR diabetic OR prediabetes OR prediabetic OR pre-diabetes OR pre-diabetic OR impaired glucose).ti.

191318 AND (information OR education OR communication).ti,ab. AND (diabetes OR diabetic OR prediabetes OR prediabetic OR pre-diabetes OR pre-diabetic OR impaired glucose).ti,ab.

13,294 ((patient OR patient-centered) ADJ1 (need OR needs OR seek* OR search* OR demand OR desire OR preference* OR wish OR wishes OR provision* OR expectation*)).ti,ab.

$2152 \quad 20$ AND (diabetes OR diabetic OR prediabetes OR prediabetic OR pre-diabetes OR pre-diabetic OR impaired glucose).ti. AND (need OR needs OR preference*).ti.

18 AND (facilitat* OR barrier* OR pitfall*).ti. AND (diabetes OR diabetic OR prediabetes OR prediabetic OR pre-diabetes OR pre-diabetic OR impaired glucose).ti,ab. question* OR asking OR ask OR seek* OR search* OR demand $O R$ desire $O R$ request* $O R$ call OR requirement $\mathrm{OR}$ requiring $\mathrm{OR}$ preference $\left.{ }^{*}\right)$ ).ti.

\begin{tabular}{|c|c|c|}
\hline 24 & 66 & $\begin{array}{l}23 \text { AND (diabetes OR diabetic OR prediabetes OR } \\
\text { prediabetic OR pre-diabetes OR pre-diabetic OR } \\
\text { impaired glucose).ti,ab. }\end{array}$ \\
\hline 25 & 6 & $\begin{array}{l}\text { ((identify* ADJ2 (interest* OR need OR needs OR } \\
\text { requirement OR preference*)) AND (diabetes OR } \\
\text { diabetic OR prediabetes OR prediabetic OR } \\
\text { pre-diabetes OR pre-diabetic OR impaired glucose)).ti. }\end{array}$ \\
\hline 26 & 32 & $\begin{array}{l}\text { (support* AND (interest* OR need OR needs OR } \\
\text { requirement OR preference*)).ti. AND (diabetes OR } \\
\text { diabetic OR prediabetes OR prediabetic OR pre- } \\
\text { diabetes OR pre-diabetic OR impaired glucose).ti,ab. }\end{array}$ \\
\hline 27 & 855 & $\begin{array}{l}\text { (((information OR education OR communication) } \\
\text { ADJ2 (interest* OR need OR needs OR question* OR } \\
\text { asking OR ask OR seek* OR search* OR demand OR } \\
\text { desire OR request* OR call OR requirement OR } \\
\text { requiring OR preference* OR wish OR wishes OR } \\
\text { provision* OR expectation*)) AND (diabetes OR } \\
\text { diabetic OR prediabetes OR prediabetic OR pre- } \\
\text { diabetes OR pre-diabetic OR impaired glucose)).ti,ab. }\end{array}$ \\
\hline 28 & 158 & $\begin{array}{l}27 \text { AND (interest* OR need* OR question* OR ask* } \\
\text { OR talk* OR online communi* OR message* OR seek* } \\
\text { OR search* OR demand OR desire OR request* OR } \\
\text { call OR requir* OR preference* OR wish* OR } \\
\text { perception* OR provision* OR expectation* OR } \\
\text { facilitat* OR barrier* OR pitfall*).ti. }\end{array}$ \\
\hline 29 & 440 & $\begin{array}{l}7 \text { OR } 9 \text { OR } 13 \text { OR } 14 \text { OR } 15 \text { OR } 17 \text { OR } 19 \text { OR } 21 \text { OR } \\
22 \text { OR } 24 \text { OR } 25 \text { OR } 26 \text { OR } 28\end{array}$ \\
\hline
\end{tabular}

\section{PsycINFO, Journals@OVID (OVID)}

\section{Databases: PsycINFO (1806-2014), Journals@OVID Research period: unlimited}

\begin{tabular}{|c|c|c|}
\hline $\begin{array}{l}\text { Search } \\
\text { step }\end{array}$ & Hits & Search \\
\hline 1 & 3002 & EXP INFORMATION SEEKING/ \\
\hline 2 & 15 & $\begin{array}{l}1 \text { AND (diabetes OR diabetic OR prediabetes OR } \\
\text { prediabetic OR pre-diabetes OR pre-diabetic OR } \\
\text { impaired glucose).ti,ab. }\end{array}$ \\
\hline 3 & 3027 & CLIENT EDUCATION/ \\
\hline 4 & 7 & $\begin{array}{l}3 \text { AND (diabetes OR diabetic OR prediabetes OR } \\
\text { prediabetic OR pre-diabetes OR pre-diabetic OR } \\
\text { impaired glucose).ti,ab. AND ((interest* OR need OR } \\
\text { needs OR question* OR asking OR ask OR seek* OR } \\
\text { search* OR demand OR desire OR request* OR call OR } \\
\text { requirement OR requiring OR preference* OR wish OR } \\
\text { wishes OR provision* OR expectation*) ADJ5 } \\
\text { information).ti,ab. }\end{array}$ \\
\hline 5 & 28 & $\begin{array}{l}\text { (information needs AND (diabetes OR diabetic OR } \\
\text { niddm OR iddm OR t } 2 \mathrm{dm} \text { OR } \mathrm{t} 1 \mathrm{dm} \text { OR prediabetes } \\
\text { OR prediabetic OR pre-diabetes OR pre-diabetic OR } \\
\text { impaired glucose)).ti,ab. }\end{array}$ \\
\hline 6 & 5992 & $\begin{array}{l}\text { ((education OR communication) ADJ2 (need OR needs } \\
\text { OR preference*)).ti,ab. }\end{array}$ \\
\hline 7 & 91 & $\begin{array}{l}6 \text { AND (diabetes OR diabetic OR prediabetes OR } \\
\text { prediabetic OR pre-diabetes OR pre-diabetic OR } \\
\text { impaired glucose).ti. }\end{array}$ \\
\hline 8 & 7387 & $\begin{array}{l}\text { ((patient OR patient-centered) ADJ1 (need OR needs } \\
\text { OR seek* OR search* OR demand OR desire OR }\end{array}$ \\
\hline
\end{tabular}


(Continued)

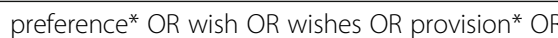
expectation*)).ti,ab.

$926 \quad 8$ AND (diabetes OR diabetic OR prediabetes OR prediabetic OR pre-diabetes OR pre-diabetic OR impaired glucose).ti. AND (need OR needs OR preference*).ti.

4494 (information AND (interest* OR need OR needs OR question* OR asking OR ask OR seek* OR search* OR demand OR desire OR request* OR call OR requirement $\mathrm{OR}$ requiring $\mathrm{OR}$ preference*)).ti.

$1130 \quad 10$ AND (diabetes OR diabetic OR prediabetes OR prediabetic OR pre-diabetes OR pre-diabetic OR impaired glucose).ti,ab.

129 ((identify* ADJ2 (interest* OR need OR needs OR requirement OR preference*)) AND (diabetes OR diabetic OR prediabetes OR prediabetic OR pre-diabetes OR pre-diabetic OR impaired glucose)).ti.

1329 (support* AND (interest* OR need OR needs OR requirement OR preference*)).ti. AND (diabetes OR diabetic OR prediabetes OR prediabetic OR pre-diabetes OR pre-diabetic OR impaired glucose).ti,ab.

509 (((information OR education OR communication) ADJ2 (interest* OR need OR needs OR question* OR asking OR ask OR seek* OR search* OR demand OR desire OR request* $O R$ call $O R$ requirement $O R$ requiring $O R$ preference* OR wish OR wishes OR provision* OR expectation*)) AND (diabetes OR diabetic OR prediabetes OR prediabetic OR pre-diabetes OR pre-diabetic OR impaired glucose)).ti,ab.

1512314 AND (interest* OR need* OR question* OR ask* OR talk* OR online communi* OR message* OR seek* OR search* OR demand OR desire OR request* OR call OR requir* OR preference* OR wish* OR perception* OR provision* OR expectation* OR facilitat* OR barrier* OR pitfall*).ti.

\section{CINAHL (EBSCO)}

Database: CINAHL (1981-2014)

Research period: unlimited

\begin{tabular}{|c|c|c|}
\hline $\begin{array}{l}\text { Search } \\
\text { step }\end{array}$ & Hits & Search \\
\hline S1 & 198 & $\begin{array}{l}\text { MW INFORMATION NEEDS AND (diabetes OR diabetic } \\
\text { OR niddm OR iddm OR t2 dm OR t1 dm OR } \\
\text { prediabetes OR prediabetic OR pre-diabetes OR } \\
\text { pre-diabetic OR impaired glucose) }\end{array}$ \\
\hline S2 & 8 & $\begin{array}{l}\text { TI information needs AND (diabetes OR diabetic OR } \\
\text { niddm OR iddm OR t2 dm OR t1 dm OR prediabetes } \\
\text { OR prediabetic OR pre-diabetes OR pre-diabetic OR } \\
\text { impaired glucose) }\end{array}$ \\
\hline S3 & 57 & $\begin{array}{l}\text { TI (education OR communication) AND TI (need OR } \\
\text { needs OR preference*) AND (diabetes OR diabetic OR } \\
\text { niddm OR iddm OR t2 dm OR t1 dm OR prediabetes } \\
\text { OR prediabetic OR pre-diabetes OR pre-diabetic OR } \\
\text { impaired glucose) }\end{array}$ \\
\hline S4 & 103 & $\begin{array}{l}\text { TI (patient OR patient-centered) AND TI (need* OR seek* } \\
\text { OR talk* OR online communi* OR message OR search* }\end{array}$ \\
\hline
\end{tabular}

(Continued)

OR demand OR desire OR preference* OR wish* OR provision* OR perception* OR expectation*) AND (diabetes OR diabetic OR niddm OR iddm OR t2 dm OR t1 dm OR prediabetes OR prediabetic OR pre-diabetes $O R$ pre-diabetic OR impaired glucose)

S5 20 TI information AND TI (interest* OR need OR needs OR question* OR asking OR ask OR seek* OR search* OR demand $O R$ desire $O R$ request* $O R$ call $O R$ requirement OR requiring OR preference*) AND (diabetes OR diabetic OR niddm OR iddm OR t2 dm OR t1 dm OR prediabetes OR prediabetic OR pre-diabetes OR pre-diabetic OR impaired glucose)

\section{The Cochrane Library (Wiley)}

\section{Databases:}

Cochrane Database of Systematic Reviews (1996-2014), Database of Abstracts of Reviews of Effects (DARE) (19942014), Cochrane Central Register of Controlled Trials (1898-2014), Cochrane Methodology Register (1904-2012), Health Technology Assessment (HTA) (1989-2014), NHS Economic Evaluation Database (NHS EED) (1968-2014) Research period: unlimited

\section{Search Hits Search}

step

\#1 0 (information need* AND (diabetes OR diabetic OR prediabetes OR prediabetic OR pre-diabetes OR pre-diabetic OR impaired glucose)):ti

\#2 4 ((education OR communication OR information) AND (need OR needs OR preference*) AND (diabetes OR diabetic OR niddm OR iddm OR t2 dm OR t1 dm OR prediabetes OR prediabetic OR pre-diabetes OR pre-diabetic OR impaired glucose)):ti

8 ((information* OR knowledge) AND (need OR needs OR needed OR seek* OR talk* OR online communi* OR search* OR demand OR desire OR preference* OR wish* OR provision* OR perception* OR expectation*) AND (diabetes OR diabetic OR niddm OR iddm OR t2dm OR t1dm OR prediabetes OR prediabetic OR pre-diabetes OR pre-diabetic OR impaired glucose)):ti question* OR asking OR ask OR seek* OR search* OR demand OR desire OR request* OR call OR requirement OR requiring OR preference*) AND (diabetes OR diabetic OR niddm OR iddm OR t2dm OR t1dm OR prediabetes OR prediabetic OR pre-diabetes OR pre-diabetic OR impaired glucose)):ti

32 ((need OR needs OR seek* OR search* OR demand OR desire OR preference* OR wish OR wishes OR provision* OR expectation*) AND (diabetes OR diabetic OR prediabetes OR prediabetic OR pre-diabetes OR pre-diabetic OR impaired glucose) AND (information OR education OR support)):ti

224 (information need AND (diabetes OR diabetic OR prediabetes OR prediabetic OR pre-diabetes OR pre-diabetic OR impaired glucose)):ti,ab,kw
12 \#6 AND (information OR knowledge):ti 


\begin{tabular}{cl} 
(Continued) & \\
\hline \#5 41 & $\# 1$ OR \#2 OR \#3 OR \#4 OR \#5 OR \#7 \\
& Cochrane Reviews:1 \\
& Other Reviews:14 \\
& Trials:12 \\
& Methods Studies:Methods Studie4 \\
& Technology Assessments:0 \\
& Economic Evaluations:10 \\
& Cochrane Groups:0 \\
\hline
\end{tabular}

Web of Science (Thomson Reuters)

Database: Web of Science (1950-2014)

Research period: unlimited

\begin{tabular}{|c|c|c|}
\hline $\begin{array}{l}\text { Search } \\
\text { step }\end{array}$ & Hits & Search \\
\hline 1 & 123 & $\begin{array}{l}\text { TITLE: (information AND (interest* OR need OR needs OR } \\
\text { question* OR talk* OR ask* OR online communi* OR seek* } \\
\text { OR search* OR demand OR desire OR request* OR call OR } \\
\text { requir* OR perception* OR preference*) AND (diabetes OR } \\
\text { diabetic OR prediabetes OR prediabetic OR pre-diabetes } \\
\text { OR pre-diabetic OR impaired glucose)) ORTITLE: ((need OR } \\
\text { needs OR seek OR search* OR demand OR desire OR } \\
\text { preference }{ }^{*} \text { OR wish OR wishes OR provision* OR } \\
\text { expectation*) AND (diabetes OR diabetic OR prediabetes } \\
\text { OR prediabetic OR pre-diabetes OR pre-diabetic OR } \\
\text { impaired glucose) AND (information OR education OR } \\
\text { support OR perception*)) ORTITLE: } \\
\text { ((education OR communication) AND (need OR } \\
\text { needs OR preference*) AND (diabetes OR diabetic OR } \\
\text { prediabetes OR prediabetic OR pre-diabetes OR } \\
\text { pre-diabetic OR impaired glucose)) } \\
\text { Timespan = All Years } \\
\text { Lemmatization = On }\end{array}$ \\
\hline
\end{tabular}

ERIC (Institute of Education Sciences)

Database: ERIC (1966-2014)

Research period: unlimited

\begin{tabular}{lll}
\hline Search step & Hits & Search \\
1 & 6 & "INFORMATION NEED*" AND "DIABETES" \\
\hline
\end{tabular}

\section{ScienceDirect (Elsevier)}

Database: ScienceDirect (Elsevier, Segmente 'Decision Sciences, Medicine,' 'Medicine and Dentistry,' 'Neuroscience,' 'Nursing and Health professions,' 'Pharmacology, Toxicology and Pharmaceutical Science,' 'Psychology', 'Social Sciences') (1823-2014)

Research period: unlimited

\begin{tabular}{lll}
\hline Search step & Hits & Search \\
1 & 9 & TITLE((information) AND (diabetes OR diabetic)) AND \\
& TITLE-ABSTR-KEY((interest* OR need OR needs OR \\
& question* OR ask* OR talk* OR online communi* OR \\
& seek* OR search* OR demand OR desire OR request* \\
& OR call OR requir* OR perception* OR preference*))
\end{tabular}

CCMed, Deutsches Ärzteblatt (DIMDI)

Database: CCMed (2001-2014), Deutsches Ärzteblatt (1996-2014)

Research period: unlimited

\begin{tabular}{|c|c|c|}
\hline $\begin{array}{l}\text { Search } \\
\text { step }\end{array}$ & Hits & Search \\
\hline 1 & 3 & $\begin{array}{l}\text { FT = (diabetes; diabetic; pre-diabet?; prediabet?; } \\
\text { impaired glucose) AND TI = (information?; education; } \\
\text { knowledge)) AND FT = (interest?; need?; question?; talk?; } \\
\text { online communi?; ask?; seek?; search?; demand; desire; } \\
\text { request?; call; requir?; perception?; preference?) }\end{array}$ \\
\hline 2 & 9 & $\begin{array}{l}\mathrm{FT}=\text { (diabetes; diabetic; pre-diabet?; prediabet?; } \\
\text { impaired glucose) AND FT = informationsbedarf }\end{array}$ \\
\hline 3 & 12 & $1 \mathrm{OR} 2$ \\
\hline
\end{tabular}

Karlsruher virtueller Katalog (Karlsruher Institut für Technologie)

Database: Karlsruher virtueller Katalog (DIMDI)

Research period: unlimited

\begin{tabular}{|c|c|c|}
\hline Search step & Hits $^{1}$ & Search \\
\hline 1 & 0 & $\begin{array}{l}\text { information need* AND (diabet* OR pre-diabet* OR } \\
\text { prediabet*) }^{*} \text { ) }\end{array}$ \\
\hline 2 & 0 & $\begin{array}{l}\text { informationsbedarf AND (diabet* OR pre-diabet* OR } \\
\text { prediabet*) }^{*} \text {. }\end{array}$ \\
\hline 3 & 0 & $\begin{array}{l}\text { preferences AND (diabet* OR pre-diabet* OR } \\
\text { prediabet }^{*} \text { ) }\end{array}$ \\
\hline
\end{tabular}

${ }^{1}$ After screening

\section{Appendix 2 \\ Search terms (Update 26/06/2015) [11] MEDLINE (OVID)}

Database: MEDLINE (1946-2015)

Research period: 10/06/2014-01/07/2015

\begin{tabular}{lll}
\hline $\begin{array}{l}\text { Search } \\
\text { step }\end{array}$ & Hits & Search \\
1 & 896 & EXP INFORMATION SEEKING BEHAVIOR/ \\
2 & 2260 & EXP INFORMATION LITERACY/ \\
3 & 4241 & EXP CONSUMER HEALTH INFORMATION/ \\
4 & 72,616 & EXP PATIENT EDUCATION AS TOPIC/ \\
5 & 782 & EXP HEALTH COMMUNICATION/ \\
6 & 1652 & 1 OR 5 \\
7 & 35 & $\begin{array}{l}\text { 6 AND (diabetes OR diabetic OR niddm OR iddm OR } \\
\end{array}$ \\
8 & 4349 & t2 dm OR t1 dm OR prediabetes OR prediabetic OR \\
9 & 87 & 8 AND (diabetes OR diabetic OR niddm OR iddm OR \\
& & t2 dm OR t1 dm OR prediabetes OR prediabetic OR
\end{tabular}


(Continued)

pre-diabetes OR pre-diabetic OR impaired glucose)

.ti,ab. AND information.ti,ab.

$10108 \quad 4$ AND ((diabetes OR diabetic OR niddm OR iddm OR t2 dm OR t1 dm OR prediabetes OR prediabetic OR pre-diabetes OR pre-diabetic OR impaired glucose).ti,ab. AND ((interest* OR need OR needs OR question* OR asking OR ask OR seek* OR search* OR demand OR desire OR request* OR call OR requirement $O R$ requiring $O R$ preference* $O R$ wish OR wishes OR provision* OR expectation*) ADJ5 information)).ti,ab.

1147 (information needs AND (diabetes OR diabetic OR niddm OR iddm OR t2 dm OR $11 \mathrm{dm}$ OR prediabetes OR prediabetic OR pre-diabetes OR pre-diabetic OR impaired glucose)).ti,ab.

124253 ((education OR communication) ADJ2 (need OR needs OR preference*)).ti,ab.

138812 AND (diabetes OR diabetic OR prediabetes OR prediabetic OR pre-diabetes OR pre-diabetic OR impaired glucose).ti.

14

10,638 ((patient OR patient-centered) ADJ1 (need OR needs OR seek* OR search* OR demand OR desire OR preference* OR wish OR wishes OR provision* OR expectation*)).ti,ab.

$1743 \quad 16$ AND (diabetes OR diabetic OR prediabetes OR prediabetic OR pre-diabetes OR pre-diabetic OR impaired glucose).ti. AND (need OR needs OR preference $\left.{ }^{*}\right)$.ti.

18314 AND (facilitat* OR barrier* OR pitfall*).ti. AND (diabetes OR diabetic OR prediabetes OR prediabetic OR pre-diabetes OR pre-diabetic OR impaired glucose).ti,ab.

1051 (((information OR education OR communication) ADJ3 (interest* OR need OR needs OR question* OR asking OR ask OR seek* OR search* OR demand $O R$ desire $O R$ request* $O R$ call $O R$ requirement $O R$ requiring $O R$ preference* $O R$ wish $O R$ wishes $O R$ provision* OR expectation*)) AND (diabetes OR diabetic OR prediabetes OR prediabetic OR pre-diabetes OR pre-diabetic OR impaired glucose)). ti,ab.

19419 AND (interest* OR need* OR question* OR ask* OR talk* OR online communi* OR message* OR seek* OR search* OR demand OR desire OR request* OR cal OR requir* OR preference* OR wish* OR perception* OR provision* OR expectation* OR facilitat* OR barrier* OR pitfall*).ti.

4256 (information AND (interest* OR need OR needs OR question* OR asking OR ask OR seek* OR search* OR demand OR desire OR request* OR call OR requirement $O R$ requiring $O R$ preference*)).ti.

21 AND (diabetes OR diabetic OR prediabetes OR prediabetic OR pre-diabetes OR pre-diabetic OR impaired glucose).ti,ab.
(Continued)

\begin{tabular}{|c|c|c|}
\hline & & $\begin{array}{l}\text { pre-diabetes OR pre-diabetic OR impaired } \\
\text { glucose)).ti. }\end{array}$ \\
\hline 24 & 22 & $\begin{array}{l}\text { (support* AND (interest* OR need OR needs OR } \\
\text { requirement OR preference*)).ti. AND (diabetes } \\
\text { OR diabetic OR prediabetes OR prediabetic OR } \\
\text { pre-diabetes OR pre-diabetic OR impaired glucose).ti,ab. }\end{array}$ \\
\hline 25 & 541 & $\begin{array}{l}7 \text { OR } 9 \text { OR } 10 \text { OR } 11 \text { OR } 13 \text { OR } 15 \text { OR } 17 \text { OR } 18 \text { OR } 20 \\
\text { OR } 22 \text { OR } 23 \text { OR } 24\end{array}$ \\
\hline 26 & 50 & limit 25 to ed="20140610-20150701" \\
\hline
\end{tabular}

\section{EMBASE (OVID)}

Database: EMBASE (1974-2015)

Research period: 24. CW 2014-27. CW 2015

\begin{tabular}{|c|c|c|}
\hline $\begin{array}{l}\text { Search } \\
\text { step }\end{array}$ & Hits & Search \\
\hline 1 & 1371 & EXP INFORMATION SEEKING/ \\
\hline 2 & 238 & EXP INFORMATION LITERACY/ \\
\hline 3 & 2647 & EXP CONSUMER HEALTH INFORMATION/ \\
\hline 4 & 25,038 & *PATIENT EDUCATION/ \\
\hline 5 & 7879 & *MEDICAL INFORMATION/ \\
\hline 6 & 102 & 1 AND 5 \\
\hline 7 & 2 & $\begin{array}{l}6 \text { AND (diabetes OR diabetic OR prediabetes OR } \\
\text { prediabetic OR pre-diabetes OR pre-diabetic OR } \\
\text { impaired glucose).ti,ab. }\end{array}$ \\
\hline 8 & 1595 & $1 \mathrm{OR} 2$ \\
\hline 9 & 29 & $\begin{array}{l}8 \text { AND (diabetes OR diabetic OR prediabetes OR } \\
\text { prediabetic OR pre-diabetes OR pre-diabetic OR } \\
\text { impaired glucose).ti,ab. }\end{array}$ \\
\hline 10 & 40 & $\begin{array}{l}3 \text { AND (diabetes OR diabetic OR prediabetes OR } \\
\text { prediabetic OR pre-diabetes OR pre-diabetic OR } \\
\text { impaired glucose).ti,ab. AND (interest* OR need OR } \\
\text { needs OR question* OR asking OR ask OR seek* OR } \\
\text { search* OR demand OR desire OR request* OR call } \\
\text { OR requirement OR requiring OR preference* OR wish } \\
\text { OR wishes OR provision* OR expectation*).ti,ab. }\end{array}$ \\
\hline 11 & 69 & $\begin{array}{l}5 \text { AND (diabetes OR diabetic OR prediabetes OR } \\
\text { prediabetic OR pre-diabetes OR pre-diabetic OR } \\
\text { impaired glucose).ti,ab. AND (interest* OR need OR } \\
\text { needs OR question* OR asking OR ask OR seek* OR } \\
\text { search* OR demand OR desire OR request* OR call } \\
\text { OR requirement OR requiring OR preference* OR wish } \\
\text { OR wishes OR provision* OR expectation*).ti,ab. }\end{array}$ \\
\hline 12 & 108 & 10 OR 11 \\
\hline 13 & 25 & $\begin{array}{l}12 \text { AND ((information OR education OR support) } \\
\text { ADJ2 (interest* OR need OR needs OR question* OR } \\
\text { asking OR ask OR seek* OR search* OR demand OR } \\
\text { desire OR request* OR call OR requirement OR } \\
\text { requiring OR preference* OR wish OR wishes OR } \\
\text { provision* OR expectation*)).ti,ab. }\end{array}$ \\
\hline 14 & 44 & $\begin{array}{l}4 \text { AND ((diabetes OR diabetic OR niddm OR iddm } \\
\text { OR t2dm OR t1dm OR prediabetes OR prediabetic } \\
\text { OR pre-diabetes OR pre-diabetic OR impaired } \\
\text { glucose).tiab. AND ((interest* OR need OR needs } \\
\text { OR question* OR asking OR ask OR seek* OR search* } \\
\text { OR demand OR desire OR request* OR call OR }\end{array}$ \\
\hline
\end{tabular}


(Continued)

requirement $O R$ requiring $O R$ preference* $O R$ wish OR wishes OR provision* OR expectation*) ADJ5 information)).ti,ab.

69 (information needs AND (diabetes OR diabetic OR niddm OR iddm OR t2dm OR t1dm OR prediabetes OR prediabetic OR pre-diabetes OR pre-diabetic OR impaired glucose)).ti,ab.

16

5502 ((education OR communication) ADJ2 (need OR needs OR preference*)).ti,ab.

1714916 AND (diabetes OR diabetic OR prediabetes OR prediabetic OR pre-diabetes OR pre-diabetic OR impaired glucose).ti.

1877 *PATIENT PREFERENCE/

1918 AND (information OR education OR communication) .ti,ab. AND (diabetes OR diabetic OR prediabetes OR prediabetic OR pre-diabetes OR pre-diabetic OR impaired glucose).ti,ab.

15113 ((patient OR patient-centered) ADJ1 (need OR needs OR seek* OR search* OR demand OR desire OR preference* OR wish OR wishes OR provision* OR expectation*)).ti,ab.

6420 AND (diabetes OR diabetic OR prediabetes OR prediabetic OR pre-diabetes OR pre-diabetic OR impaired glucose).ti. AND (need OR needs OR preference*).ti.

$22 \quad 218$ AND (facilitat* OR barrier* OR pitfall*).ti. AND (diabetes OR diabetic OR prediabetes OR prediabetic OR pre-diabetes OR pre-diabetic OR impaired glucose).ti,ab.

5002 (information AND (interest* OR need OR needs OR question* OR asking OR ask OR seek* OR search* OR demand OR desire OR request* OR call OR requirement $\mathrm{OR}$ requiring $\mathrm{OR}$ preference*)).ti.

$24 \quad 7123$ AND (diabetes OR diabetic OR prediabetes OR prediabetic OR pre-diabetes OR pre-diabetic OR impaired glucose).ti,ab.

((identify* ADJ2 (interest* OR need OR needs OR requirement OR preference*)) AND (diabetes OR diabetic OR prediabetes OR prediabetic OR pre-diabetes OR pre-diabetic OR impaired glucose)).ti.

(support* AND (interest* OR need OR needs OR requirement OR preference*)).ti. AND (diabetes OR diabetic OR prediabetes OR prediabetic OR pre-diabetes OR pre-diabetic OR impaired glucose).ti,ab.

984 (((information OR education OR communication) ADJ2 (interest* OR need OR needs OR question* OR asking OR ask OR seek* OR search* OR demand OR desire OR request* $O R$ call $O R$ requirement $O R$ requiring $O R$ preference* OR wish OR wishes OR provision* OR expectation*)) AND (diabetes OR diabetic OR prediabetes OR prediabetic OR pre-diabetes OR prediabetic OR impaired glucose)).ti,ab. search* OR demand OR desire OR request* OR call OR requir* OR preference* OR wish* OR perception* OR provision* OR expectation* OR facilitat* OR barrier* OR pitfall*).ti. 22 OR 24 OR 25 OR 26 OR 28

\section{PsycINFO, Journals@OVID (OVID)}

Database: PsycINFO (1806-2015) Journals@OVID

Research period: 10/06/2014-01/07/2015 (PsycINFO) and publication year 2014-2015 (Journals@Ovid)

\begin{tabular}{|c|c|c|}
\hline $\begin{array}{l}\text { Search } \\
\text { step }\end{array}$ & Hits & Search \\
\hline 1 & 3175 & EXP INFORMATION SEEKING/ \\
\hline 2 & 19 & $\begin{array}{l}1 \text { AND (diabetes OR diabetic OR prediabetes OR } \\
\text { prediabetic OR pre-diabetes OR pre-diabetic OR } \\
\text { impaired glucose).ti,ab. }\end{array}$ \\
\hline 3 & 3209 & CLIENT EDUCATION/ \\
\hline 4 & 10 & $\begin{array}{l}3 \text { AND (diabetes OR diabetic OR prediabetes OR } \\
\text { prediabetic OR pre-diabetes OR pre-diabetic OR } \\
\text { impaired glucose).ti,ab. AND ((interest* OR need OR } \\
\text { needs OR question* OR asking OR ask OR seek* OR } \\
\text { search* OR demand OR desire OR request* OR call } \\
\text { OR requirement OR requiring OR preference* OR } \\
\text { wish OR wishes OR provision* OR expectation*) } \\
\text { ADJ5 information).ti,ab. }\end{array}$ \\
\hline 5 & 30 & $\begin{array}{l}\text { (information needs AND (diabetes OR diabetic OR } \\
\text { niddm OR iddm OR t2dm OR t } 1 d m \text { OR prediabetes } \\
\text { OR prediabetic OR pre-diabetes OR pre-diabetic OR } \\
\text { impaired glucose)).ti,ab. }\end{array}$ \\
\hline 6 & 6616 & $\begin{array}{l}\text { ((education OR communication) ADJ2 (need OR } \\
\text { needs OR preference*)).ti,ab. }\end{array}$ \\
\hline 7 & 100 & $\begin{array}{l}6 \text { AND (diabetes OR diabetic OR prediabetes OR } \\
\text { prediabetic OR pre-diabetes OR pre-diabetic OR } \\
\text { impaired glucose).ti. }\end{array}$ \\
\hline 8 & 8087 & $\begin{array}{l}\text { ((patient OR patient-centered) ADJ1 (need OR } \\
\text { needs OR seek* OR search* OR demand OR desire } \\
\text { OR preference }{ }^{*} \text { OR wish OR wishes OR provision* } \\
\text { OR expectation*)).ti,ab. }\end{array}$ \\
\hline 9 & 29 & $\begin{array}{l}8 \text { AND (diabetes OR diabetic OR prediabetes OR } \\
\text { prediabetic OR pre-diabetes OR pre-diabetic OR } \\
\text { impaired glucose).ti. AND (need OR needs OR } \\
\text { preference*).ti. }\end{array}$ \\
\hline
\end{tabular}

4835 (information AND (interest* OR need OR needs OR question* OR asking OR ask OR seek* OR search* OR demand OR desire OR request* OR call OR requirement $O R$ requiring $O R$ preference $\left.{ }^{*}\right)$ ).ti.

3510 AND (diabetes OR diabetic OR prediabetes OR prediabetic OR pre-diabetes OR pre-diabetic OR impaired glucose).ti,ab.

9 ((identify* ADJ2 (interest* OR need OR needs OR requirement $O R$ preference*)) AND (diabetes $O R$ diabetic OR prediabetes OR prediabetic OR pre-diabetes OR pre-diabetic OR impaired glucose)).ti.

38 (support* AND (interest* OR need OR needs OR requirement OR preference*)).ti. AND (diabetes OR diabetic OR prediabetes OR prediabetic OR pre-diabetes $O R$ pre-diabetic OR impaired glucose).ti,ab.

572 (((information OR education OR communication) ADJ2 (interest* OR need OR needs OR question* OR asking OR ask OR seek* OR search* OR demand $O R$ desire $O R$ request* $O R$ call $O R$ requirement $O R$ requiring $O R$ preference* $O R$ wish $O R$ wishes $O R$ provision* OR expectation*)) AND (diabetes OR diabetic OR prediabetes OR prediabetic OR prediabetes OR pre-diabetic OR impaired glucose)).ti,ab. 


\begin{tabular}{|c|c|c|}
\hline 15 & 136 & $\begin{array}{l}14 \text { AND (interest* OR need* OR question* OR ask* OR } \\
\text { talk* OR online communi* OR message* OR seek* OR } \\
\text { search* OR demand OR desire OR request* OR call } \\
\text { OR requir* OR preference* OR wish* OR perception* } \\
\text { OR provision* OR expectation* OR facilitat* OR } \\
\text { barrier* OR pitfall*).ti. }\end{array}$ \\
\hline 16 & 294 & 2 OR 4 OR 5 OR 7 OR 9 OR 11 OR 12 OR 13 OR 15 \\
\hline 17 & 210 & limit 16 to up="20140610-20150701" \\
\hline 18 & 209 & remove duplicates from 17 \\
\hline 19 & 57 & $\begin{array}{l}\text { limit } 18 \text { to yr="2013 - 2015" } \\
\text { Journals@Ovid: } 43 \\
\text { Psyclnfo: } 14\end{array}$ \\
\hline
\end{tabular}

\section{CINAHL (EBSCO)}

Database: CINAHL (1981-2015)

Research period: 01/06/2014-31/07/2015

\begin{tabular}{|c|c|c|}
\hline Search step & Hits & Search \\
\hline S1 & 206 & $\begin{array}{l}\text { MW INFORMATION NEEDS AND (diabetes OR diabetic } \\
\text { OR niddm OR iddm OR t2 dm OR t1 dm OR } \\
\text { prediabetes OR prediabetic OR pre-diabetes OR } \\
\text { pre-diabetic OR impaired glucose) }\end{array}$ \\
\hline S2 & 7 & $\begin{array}{l}\text { TI information needs AND (diabetes OR diabetic } \\
\text { OR niddm OR iddm OR } \mathrm{t} 2 \mathrm{dm} \text { OR } \mathrm{t} 1 \mathrm{dm} \text { OR } \\
\text { prediabetes OR prediabetic OR pre-diabetes } \\
\text { OR pre-diabetic OR impaired glucose) }\end{array}$ \\
\hline S3 & 51 & $\begin{array}{l}\text { TI (education OR communication) AND TI (need } \\
\text { OR needs OR preference*) AND (diabetes OR } \\
\text { diabetic OR niddm OR iddm OR t2 dm OR t1 dm } \\
\text { OR prediabetes OR prediabetic OR pre-diabetes } \\
\text { OR pre-diabetic OR impaired glucose) }\end{array}$ \\
\hline S4 & 110 & $\begin{array}{l}\text { TI (patient OR patient-centered) AND TI (need* OR } \\
\text { seek* OR talk* OR online communi* OR message } \\
\text { OR search* OR demand OR desire OR preference* } \\
\text { OR wish* OR provision* OR perception* OR } \\
\text { expectation*) AND (diabetes OR diabetic OR niddm } \\
\text { OR iddm OR t2 dm OR t1 dm OR prediabetes OR } \\
\text { prediabetic OR pre-diabetes OR pre-diabetic OR } \\
\text { impaired glucose) }\end{array}$ \\
\hline S5 & 22 & $\begin{array}{l}\text { TI information AND TI (interest* OR need OR needs } \\
\text { OR question* OR asking OR ask OR seek* OR search* } \\
\text { OR demand OR desire OR request* OR call OR } \\
\text { requirement OR requiring OR preference*) AND } \\
\text { (diabetes OR diabetic OR niddm OR iddm OR t2 dm } \\
\text { OR t1 dm OR prediabetes OR prediabetic OR } \\
\text { pre-diabetes OR pre-diabetic OR impaired glucose) }\end{array}$ \\
\hline S6 & 372 & S1 OR S2 OR S3 OR S4 OR S5 \\
\hline S7 & 21 & (EM 20140601-20150731) AND S6 \\
\hline
\end{tabular}

\section{The Cochrane Library (Wiley)}

Databases: Cochrane Database of Systematic Reviews (1996-2015), Database of Abstracts of Reviews of Effects (DARE) (1994-2015), Cochrane Central Register of Controlled Trials (1898-2015), Cochrane Methodology Register (1904-2012), Health Technology
Assessment (HTA) (1989-2015), NHS Economic Evaluation Database (NHS EED) (1968-2015)

Research period: 06/2014-06/2015

\begin{tabular}{|c|c|c|}
\hline $\begin{array}{l}\text { Search } \\
\text { step }\end{array}$ & Hits & Search \\
\hline$\# 1$ & 0 & $\begin{array}{l}\text { (information need* AND (diabetes OR diabetic } \\
\text { OR prediabetes OR prediabetic OR pre-diabetes } \\
\text { OR pre-diabetic OR impaired glucose)):ti }\end{array}$ \\
\hline \#2 & 4 & $\begin{array}{l}\text { ((education OR communication OR information) } \\
\text { AND (need OR needs OR preference*) AND } \\
\text { (diabetes OR diabetic OR niddm OR iddm OR t2 } \\
\text { dm OR t1 dm OR prediabetes OR prediabetic } \\
\text { OR pre-diabetes OR pre-diabetic OR impaired } \\
\text { glucose)):ti }\end{array}$ \\
\hline \#3 & 8 & $\begin{array}{l}\text { ((information* OR knowledge) AND (need OR needs } \\
\text { OR needed OR seek* OR talk* OR online communi* } \\
\text { OR search* OR demand OR desire OR preference* } \\
\text { OR wish* OR provision* OR perception* OR expectation*) } \\
\text { AND (diabetes OR diabetic OR niddm OR iddm OR t2 } \\
\text { dm OR t1 dm OR prediabetes OR prediabetic OR } \\
\text { pre-diabetes OR pre-diabetic OR impaired glucose)):ti }\end{array}$ \\
\hline \#4 & 3 & $\begin{array}{l}\text { (information AND (interest* OR need OR needs OR } \\
\text { question* OR asking OR ask OR seek* OR search* } \\
\text { OR demand OR desire OR request* OR call OR } \\
\text { requirement OR requiring OR preference*) AND } \\
\text { (diabetes OR diabetic OR niddm OR iddm OR t2 dm } \\
\text { OR t1 dm OR prediabetes OR prediabetic OR } \\
\text { pre-diabetes OR pre-diabetic OR impaired glucose)):ti }\end{array}$ \\
\hline \#5 & 40 & $\begin{array}{l}\text { ((need OR needs OR seek* OR search* OR demand } \\
\text { OR desire OR preference* OR wish OR wishes OR } \\
\text { provision* OR expectation*) AND (diabetes OR } \\
\text { diabetic OR prediabetes OR prediabetic OR } \\
\text { pre-diabetes OR pre-diabetic OR impaired glucose) } \\
\text { AND (information OR education OR support)):ti }\end{array}$ \\
\hline \#6 & 117 & $\begin{array}{l}\text { (information need AND (diabetes OR diabetic } \\
\text { OR prediabetes OR prediabetic OR pre-diabetes } \\
\text { OR pre-diabetic OR impaired glucose)):ti,ab,kw }\end{array}$ \\
\hline \#7 & 7 & \#6 AND (information OR knowledge):ti \\
\hline \#5 & 46 & $\begin{array}{l}\text { \#1 OR \#2 OR \#3 OR \#4 OR \#5 OR \#7 } \\
\text { Cochrane Reviews:1 } \\
\text { Other Reviews:20 } \\
\text { Trials:10 } \\
\text { Methods Studies:2 } \\
\text { Technology Assessments:0 } \\
\text { Economic Evaluations:13 } \\
\text { Cochrane Groups:0 }\end{array}$ \\
\hline \#6 & 0 & $\begin{array}{l}\# 1 \mathrm{OR} \# 2 \mathrm{OR} \# 3 \mathrm{OR} \# 4 \text { OR \#5 OR \#7Online Publication } \\
\text { Date in the last } 12 \text { months }\end{array}$ \\
\hline
\end{tabular}

\section{Web of Science (Thomson Reuters)}

Database: Web of Science (1950-2015)

Research period: publication year 2014-2015

$$
\begin{array}{lll}
\hline \begin{array}{l}
\text { Search } \\
\text { step }
\end{array} & \text { Hits } & \text { Search } \\
1 & 163 \begin{array}{l}
\text { TITLE: (information AND (interest* OR need OR } \\
\text { needs OR question* OR talk* OR ask* OR online }
\end{array} \\
& \begin{array}{l}
\text { communi* OR seek* OR search* OR demand OR } \\
\text { desire OR request* OR call OR requir* OR perception* }
\end{array}
\end{array}
$$


(Continued)

OR preference*) AND (diabetes OR diabetic OR prediabetes OR prediabetic OR pre-diabetes OR pre-diabetic OR impaired glucose)) ORTITLE: ((need OR needs OR seek* OR search* OR demand OR desire OR preference* OR wish OR wishes OR provision* OR expectation*) AND (diabetes OR diabetic OR prediabetes OR prediabetic OR pre-diabetes OR pre-diabetic OR impaired glucose)

AND (information OR education OR support OR perception*)) ORTITLE: ((education OR communication) AND (need OR needs OR preference*) AND (diabetes OR diabetic OR prediabetes OR prediabetic OR pre-diabetes OR pre-diabetic OR impaired glucose)) Timespan $=$ All Years Lemmatization $=$ On OR question* OR talk* OR ask* OR online communi* OR seek* OR search* OR demand OR desire OR request* OR call OR requir* OR perception* OR preference*) AND (diabetes OR diabetic OR prediabetes OR prediabetic OR pre-diabetes OR pre-diabetic OR impaired glucose))) OR TITLE: (( (need OR needs OR seek* OR search* OR demand $O R$ desire OR preference* OR wish OR wishes OR provision* OR expectation*) AND (diabetes OR diabetic OR prediabetes OR prediabetic OR pre-diabetes OR pre-diabetic OR impaired glucose) AND (information OR education OR support OR perception*))) OR TITLE: (((education OR communication) AND (need OR needs OR preference*) AND (diabetes OR diabetic OR prediabetes OR prediabetic OR pre-diabetes OR pre-diabetic OR impaired glucose))) Refined by: PUBLICATION YEARS: (2015 OR 2014) Timespan = All years Search language $=$ Auto

\section{ERIC (Institute of Education Sciences)}

Database: ERIC (1966-2015)

Research period: unlimited.

\begin{tabular}{lll}
\hline Search step & Hits & Search \\
1 & 7 & "INFORMATION NEED*" AND "DIABETES" \\
\hline
\end{tabular}

Download: 1 data set

\section{ScienceDirect (Elsevier)}

Database: ScienceDirect (Elsevier, Segmente 'Decision Sciences, Medicine,' 'Medicine and Dentistry,' 'Neuroscience,' 'Nursing and Health professions,' 'Pharmacology, Toxicology and Pharmaceutical Science, 'Psychology, 'Social Sciences') (1823-2015)

Research period: Publication year 2014-2015

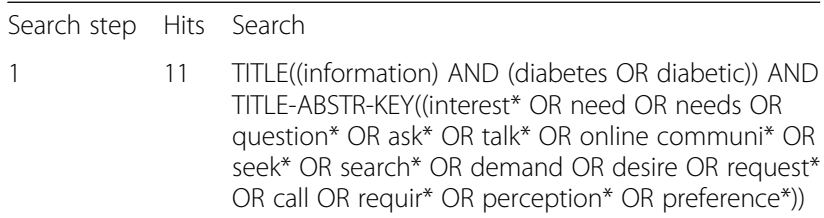

\begin{tabular}{|c|c|c|}
\hline 2 & 4 & $\begin{array}{l}\text { pub-date }>2013 \text { and TITLE((information) AND } \\
\text { (diabetes OR diabetic)) AND TITLE-ABSTR-KEY } \\
\text { ((interest* OR need OR needs OR question* OR } \\
\text { ask }^{*} \text { OR talk* OR online communi* OR seek* OR } \\
\text { search* OR demand OR desire OR request* OR } \\
\left.\text { call OR requir* OR perception* OR preference }{ }^{*}\right) \text { ) }\end{array}$ \\
\hline
\end{tabular}

\section{CCMed (MedPilot, ZBMed)}

Database: CCMed (2001-2015)

Research period: Publication year 2014-2015

\begin{tabular}{|c|c|c|}
\hline Search step & Hits & Search \\
\hline 1 & 37 & $\begin{array}{l}\text { TI = ((diabetes OR diabetic OR pre-diabet* OR } \\
\text { prediabet* OR impaired glucose) AND (interest* } \\
\text { OR need* OR question* OR talk* OR online } \\
\text { communi* OR ask* OR seek* OR search* OR } \\
\text { demand OR desire OR request* OR call OR requir* } \\
\text { OR perception* OR preference*)) AND TI = } \\
\text { ((information* OR education OR knowledge)) } \\
\text { Jahre 2014-2015: } 1\end{array}$ \\
\hline 2 & 392 & $\begin{array}{l}\mathrm{TI}=((\text { diabetes OR diabetic OR pre-diabet* OR } \\
\text { prediabet* OR impaired glucose)) AND TI = } \\
\text { ((information* OR education OR knowledge)) } \\
\text { Jahre 2014-2015: } 21\end{array}$ \\
\hline
\end{tabular}

Download: 22 datasets

\section{Deutsches Ärzteblatt (Deutscher Ärzteverlag)}

Database: Deutsches Ärzteblatt (1996-2015)

Research period: Publication year 2014-2015

\begin{tabular}{lll}
\hline $\begin{array}{l}\text { Search } \\
\text { step }\end{array}$ & Hits & Search \\
1 & 0 & $\begin{array}{l}\text { Diabetes informationsbedarfPublicationyear 2014- } \\
2015\end{array}$ \\
2 & 0 & Diabetes information needPublicationyear 2014-2015 \\
\hline
\end{tabular}

\section{Karlsruher virtueller Katalog (Karlsruher Institut für Technologie)}

Database: Karlsruher virtueller Katalog (DIMDI)

Research period: unlimited

\begin{tabular}{lll}
\hline Search step & Hits $^{1}$ & $\begin{array}{l}\text { Search } \\
1\end{array}$ \\
0 & $\begin{array}{l}\text { information need* AND (diabet* OR pre-diabet* } \\
\text { OR prediabet*) }\end{array}$ \\
3 & 0 & $\begin{array}{l}\text { informationsbedarf AND (diabet* OR pre-diabet* } \\
\text { OR prediabet*) } \\
\text { preferences AND (diabet* OR pre-diabet } \\
\text { OR prediabet* }\end{array}$ \\
\hline
\end{tabular}

\footnotetext{
${ }^{1}$ After screening
} 


\section{Additional file}

Additional file 1: PRISMA (Preferred Reporting Items for Systematic review and Meta-Analysis) Checklist 2009. Checklist: recommended items to address in a systematic review. (DOC $145 \mathrm{~kb}$ )

\section{Abbreviations}

A.I.: Andrea Icks; A.S.: Astrid Stephan; DM: Diabetes mellitus; GDM: gestational diabetes; IN: Information needs; J.G.: Jutta Genz; L.B.: Lisa Biernatzki; M.R.: Michaela Ritschel; MODY: Maturity-onset diabetes of the young; S.D.: Sigrid Droste; S.K.: Silke Kuske; T1DM: Type 1 diabetes mellitus; T2DM: Type 2 diabetes mellitus

\section{Funding}

This work was supported by the Research Committee of the Heinrich Heine University [9772577].

\section{Availability of data and materials}

Not applicable

\section{Authors' contributions}

$\mathrm{Al}$ is the guarantor of this protocol. NC, SK, CB, AS, Al and LB contributed to the concept, design and drafting of the protocol. SK, SD, LB and JG participated in the development of the systematic search strategies, and $L B$, SK, JG and MR conducted the review. SK, LB, NC, MR, AS, JG, SG, NE and Al made contributions to the write-up and editing of the protocol. All authors revised critically and approved the final manuscript of the protocol.

\section{Ethics approval and consent to participate}

Not applicable

\section{Consent for publication}

Not applicable

\section{Competing interests}

The authors declare that they have no competing interests.

\section{Publisher's Note}

Springer Nature remains neutral with regard to jurisdictional claims in published maps and institutional affiliations.

\begin{abstract}
Author details
${ }^{1}$ Institute for Health Services Research and Health Economics, Centre for Health and Society, Faculty of Medicine, Heinrich Heine University, Moorenstraße 5, 40225 Düsseldorf, Germany. ${ }^{2}$ Institute for Health Services Research and Health Economics, German Diabetes Center (DDZ), Leibniz Institute for Diabetes Research, at Heinrich Heine University Düsseldorf, Auf'm Hennekamp 65, 40225 Düsseldorf, Germany. ${ }^{3}$ Center for Health Communication and Health Services Research (CHSR), Department for Psychosomatic Medicine and Psychotherapy, University Hospital Bonn, Sigmund-Freud-Str. 25, 53127 Bonn, Germany. ${ }^{4}$ German Center for Diabetes Research (DZD), Ingolstädter Landstraße 1, 85764 Neuherberg, Germany.
\end{abstract}

Received: 31 August 2016 Accepted: 17 January 2018

Published online: 14 February 2018

\section{References}

1. Mühlhauser I. Diabetes experts' reasoning about diabetes prevention studies: a questionnaire survey. BMC Research Notes. 2008;1:90.

2. Ahola AJ, Groop PH. Barriers to self-management of diabetes. Diabet Med. 2013;30:413-20.

3. Beeney $L$, Bakry AA, Dunn SM. Patient psychological and information needs when the diagnosis is diabetes. Patient Educ Couns. 1996;29:109-16.

4. Duggan C, Bates I. Medicine information needs of patients: the relationships between information needs, diagnosis and disease. Qual Saf Health Care. 2008:17:85-9.

5. Kandula S, Curtis D, Hill B, Zeng-Treitler Q. Use of topic modeling for recommending relevant education material to diabetic patients. AMIA Annu Symp Proc. 2011;10:674-82.
6. Mühlhauser I, Sawicki P, Didjurgeit U, Jörgens V, Berger M. Uncontrolled hypertension in type 1 diabetes: assessment of patients' desires about treatment and improvement of blood pressure control by a structured treatment and teaching programme. Diabet Med. 1988;5:693-8.

7. Pieper D, Jülich F, Antoine SL, Bächle C, Chernyak N, Genz J, Eikermann M, Icks A. Studies analysing the need for health-related information in Germany-a systematic review. BMC Health Serv Res. 2015;15:407.

8. St Jean BL. Information behavior of people diagnosed with a chronic serious health condition: a longitudinal study: The University of Michigan; 2012. https://deepblue.lib.umich.edu/bitstream/handle/2027.42/91570/ bstjean_1.pdf?sequence=1. Accessed 17 Oct 2017

9. St Jean BL. Devising and implementing a card-sorting technique for a longitudinal investigation of the information behavior of people with type 2 diabetes. Library \& Information Science Research. 2014;36:16-26.

10. Moher D, Liberati A, Tetzlaff J, Altman DG, PRISMA Group. Preferred reporting items for systematic reviews and meta-analyses: the PRISMA statement. PLoS Med. 2009;7:e1000097.

11. Kuske S, Schiereck T, Grobosch S, Paduch A, Droste S, Halbach S, Icks A. Diabetes-related information-seeking behaviour: a systematic review. Syst Rev. 2017;6(1).

12. Ormandy P. Defining information need in health-assimilating complex theories derived from information science. Health Expect. 2011;14:92-104.

13. Finfgeld-Connett D. Use of content analysis to conduct knowledge-building and theory-generating qualitative systematic reviews. Qual Res. 2014; https://doi.org/10.1177/1468794113481790.

14. National Institute for Health and Care Excellence. Assessing the quality of evidence. In: Methods for the development of NICE Public Health Guidance (third edition). National Institute for Health and Care Excellence (NICE); 2012. p. 72-3.

15. Pluye $P$, Gagnon MP, Griffiths F, Johnson-Lafleur J. A scoring system for appraising mixed methods research, and concomitantly appraising qualitative, quantitative and mixed methods primary studies in mixed studies reviews. Int J Nurs Stud. 2009;46:529-46.

16. Lamberts EJ, Bouvy ML, van Hulten RP. The role of the community pharmacist in fulfilling information needs of patients starting oral antidiabetics. Res Social Adm Pharm. 2010;6:354-64.

17. Lee DY, Armour C, Krass I. The development and evaluation of written medicines information for type 2 diabetes. Health Educ Res. 2007:22:918-30.

18. Ravert RD, Hancock MD, Ingersoll GM. Online forum messages posted by adolescents with type 1 diabetes. Diabetes Educ. 2003;30:827-34

19. Olsen Roper S, Call A, Leishman J, Ratcliffe GC, Mandleco BL, Dyches TT, Marshall ES. Type 1 diabetes: children and adolescents' knowledge and questions. J Adv Nurs. 2009;65:1705-14.

20. Savage S, Dabkowski S, Dunning T. The education and information needs of young adults with type 2 diabetes: a qualitative study. Journal of Nursing \& Healthcare of Chronic Illness. 2009; https://doi.org/10.1111/j. 1752-9824.2009.01035.x

21. Sparud-Lundin C, Ranerup A, Berg M. Internet use, needs and expectations of web-based information and communication in childbearing women with type 1 diabetes. BMC Med Inform Decis Mak. 2011;11:49.

22. van Esch SC, Cornel MC, Snoek FJ. "I am pregnant and my husband has diabetes. Is there a risk for my child?" A qualitative study of questions asked by email about the role of genetic susceptibility to diabetes. BMC Public Health. 2010;10:1-8.

23. Whetstone M: Adults living with type 2 diabetes: kept personal health information items as expressions of need. http://diginole.lib.fsu.edu/islandora/ object/fsu:183936/datastream/PDF/view (2013). Accessed 17 Oct 2017.

24. Whitford DL, Paul G, Smith SM. Patient generated "frequently asked questions": identifying informational needs in a RCT of peer support in type 2 diabetes. Prim Care Diabetes. 2013:7:103-9.

25. Wilson $\mathrm{V}$. Patient use of the internet for diabetes information. Nurs Times. 2013:109:18-20.

26. Goldman RE, Sanchez-Hernandez M, Ross-Degnan D, Piette JD, Trinacty CM, Simon SR. Developing an automated speech-recognition telephone diabetes intervention. Int J Qual Health Care. 2008;20:264-70

27. Hjelm K, Berntorp K, Frid A, Aberg A, Apelqvist J. Beliefs about health and illness in women managed for gestational diabetes in two organisations. Midwifery. 2008:24:168-82.

28. Lindenmeyer A, Bowyer V, Roscoe J, Dale J, Sutcliffe P. Oral health awareness and care preferences in patients with diabetes: a qualitative study. Fam Pract. 2013;30:113-8. 
29. McCorry NK, Hughes C, Spence D, Holmes VA, Harper R. Pregnancy planning and diabetes: a qualitative exploration of women's attitudes toward preconception care. J Midwifery Womens Health. 2012;57:396-402.

30. Meyfroidt S, Aeyels D, Van Audenhove C, Verlinde C, Peers J, Panella M, Vanhaecht K. How do patients with uncontrolled diabetes in the Brusselscapital region seek and use information sources for their diet? Prim Health Care Res Dev. 2013;14:229-39.

31. Peel E, Parry O, Douglas M, Lawton J. Diagnosis of type 2 diabetes: a qualitative analysis of patients' emotional reactions and views about information provision. Patient Educ Couns. 2004;53:269-75.

32. Chen AT. Exploring online support spaces: using cluster analysis to examine breast cancer, diabetes and fibromyalgia support groups. Patient Educ Couns. 2012;87:250-7.

33. Frandsen KB, Kristensen JS. Diet and lifestyle in type 2 diabetes: the patient's perspective. Practical Diabetes Intern. 2002; https://doi.org/10.1002/pdi.327.

34. Hajos TR, Polonsky WH, Twisk JW, Dain MP, Snoek FJ. Do physicians understand type 2 diabetes patients' perceptions of seriousness; the emotional impact and needs for care improvement? A cross-national survey. Patient Educ Couns. 2011;85:258-63.

35. Robertson JL, Akhtar S, Petrie JR, Brown FJ, Jones GC, Perry CG, Paterson KR. How do people with diabetes access information? Practical Diabetes. 2005; https://doi.org/10.1002/pdi.818.

36. Wilkinson $E$, Randhawa G, Singh M. Quality improvements in diabetes care, how holistic have they been? A case-study from the United Kingdom. Int J Equity Health. 2014;13:29.

37. Rutten $\sqcup$, Arora NK, Bakos AD, Aziz N, Rowland J. Information needs and sources of information among cancer patients: a systematic review of research (1980-2003). Patient Educ Couns. 2005;57:250-61.

38. World Health Organization: Global report on diabetes. http://apps.who. int/iris/bitstream/10665/204871/1/9789241565257_eng.pdf (2016). Accessed 17 Oct 2017.

39. Skinner EA, Wellborn JG. Coping during childhood and adolescence: A motivational perspective. In: Featherman DL, Lerner RM, Perlmutter M, editors. Life-span development and behavior, Vol. 12. Hillsdale; 1994. p. 91-133.

\section{Submit your next manuscript to BioMed Central and we will help you at every step:}

- We accept pre-submission inquiries

- Our selector tool helps you to find the most relevant journal

- We provide round the clock customer support

- Convenient online submission

- Thorough peer review

- Inclusion in PubMed and all major indexing services

- Maximum visibility for your research

Submit your manuscript at www.biomedcentral.com/submit 